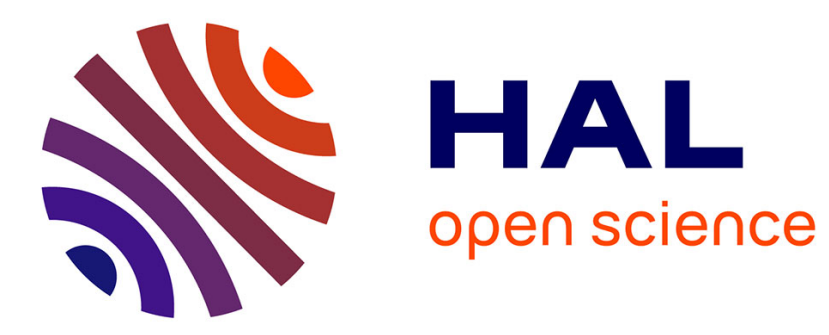

\title{
A spatial physiological model for p53 intracellular dynamics
}

Luna Dimitrio, Jean Clairambault, Roberto Natalini

\section{To cite this version:}

Luna Dimitrio, Jean Clairambault, Roberto Natalini. A spatial physiological model for p53 intracellular dynamics. 2012. hal-00726014

\section{HAL Id: hal-00726014 https://hal.inria.fr/hal-00726014}

Preprint submitted on 28 Aug 2012

HAL is a multi-disciplinary open access archive for the deposit and dissemination of scientific research documents, whether they are published or not. The documents may come from teaching and research institutions in France or abroad, or from public or private research centers.
L'archive ouverte pluridisciplinaire HAL, est destinée au dépôt et à la diffusion de documents scientifiques de niveau recherche, publiés ou non, émanant des établissements d'enseignement et de recherche français ou étrangers, des laboratoires publics ou privés. 


\title{
A spatial physiological model for p53 intracellular dynamics
}

\author{
Luna Dimitrio $^{\mathrm{a}, \mathrm{c}, *}$, Jean Clairambault ${ }^{\mathrm{a}}$, Roberto Natalini ${ }^{\mathrm{b}}$ \\ ${ }^{a}$ INRIA Paris-Rocquencourt \&3 UPMC, 4 Place Jussieu, F-75005 Paris \\ ${ }^{b}$ IAC-CNR \&3 Via dei Taurini 19, I-00185 Rome, Italy \\ ${ }^{c}$ SBAI Department, University Sapienza 63 Piazzale Aldo Moro 5, I-00185 Rome, Italy
}

\begin{abstract}
In this paper we design and analyse a physiologically based model representing the accumulation of protein p53 in the nucleus after triggering of ATM by DNA damage. The p53 protein is known to have a central role in the response of the cell to cytotoxic or radiotoxic insults resulting in DNA damage. A reasonable requirement for a model describing intracellular signalling pathways is to take into account the basic feature of eukaryotic cells: the distinction between nucleus and cytoplasm. Our aim is to show, on a simple reaction network describing p53 dynamics, how this basic distinction provides a framework which is able to yield expected oscillatory dynamics without introducing either positive feedbacks or delays in the reactions. Furthermore we prove that oscillations appear only if some spatial constraints are respected, e.g. if the diffusion coefficients correspond to known biological values. Finally we analyse how the spatial features of a cell influence the dynamic response of the p53 network to DNA damage, pointing out that the protein oscillatory dynamics is indeed a response that is robust towards changes with respect to cellular environments. Even if we change the cell shape or its volume or better its ribosomal distribution, we observe that DNA damage yields sustained oscillations of p53.
\end{abstract}

Keywords: Mathematical Models, Cellular biology, Intracellular dynamics, Partial Differential Equations, Signalling Pathways, p53

Submitted May 2012

\footnotetext{
${ }^{*}$ Corresponding author

Email addresses: luna.dimitrio@inria.fr (Luna Dimitrio), jean.clairambault@inria.fr (Jean Clairambault), roberto.natalini@cnr.it (Roberto Natalini)
} 


\section{Introduction}

Protein p53 is a tumour suppressor and its synthesis is essential for healthy cell survival. Indeed when damage to the DNA is detected, p53 blocks the cell cycle, triggers DNA repair processes or launches apoptosis of the cell. The protein p53 acts primarily as a transcription factor, so that it needs to accumulate in the nucleus to start the processes mentioned above, thus indirectly determining the cell fate, survival or apoptosis.

Experimental observations on cell populations [1] and on single cells [2] pointed out an interesting aspect of p53 dynamics: its oscillatory behaviour. While in healthy cells p53 concentration is low and at steady state, in damaged cells, where p53 is activated, the protein concentration rises and undamped oscillations appear. There are two main issues about the oscillatory behaviour of p53. The first one is to understand the biological meaning of these oscillations, i.e. how oscillations are linked to cell fate 'decision' (towards life or death). The second issue is to identify the physiological mechanisms driving the observed oscillations. Before understanding why oscillations occur, it is important to recognize the key features that generate it and mathematical modelling can help to answer this question. Such an approach allows also the analysis of p53 alterations in disease (knowing that p53 is mutated and ineffective in about $50 \%$ of cancers) and it represents a challenge with possible therapeutic implications. The aim of this work is to design and analyse a deterministic model for the evolution of the protein p53, that considers the spatial structure of the cell, in order to describe the protein intracellular dynamics. A second goal is to exhibit both a stationary behaviour of p53 intracellular concentrations in the absence of its triggering by DNA damage, and on the contrary sustained oscillations as soon as such damage is sensed by the cell.

The paper is organised as follows. In section 2, we recall biological facts about p53 and how they have been taken into account in the previous published models. In section 3 we present an Ordinary Differential Equation (ODE) compartmental model with two compartments, nucleus and cytoplasm, with exchanges between them, that exhibits the prescribed oscillations. Section 4 contains the core of this work, namely a Partial Differential Equation (PDE) model showing the desired sustained oscillations in the presence of triggering by DNA damage. We finally discuss in sections 5 and 6 the main features of this model, its limitations, and possible future extensions to include even more biological relevance.

\section{Common knowledge about protein p53}

\subsection{Biological background}

The p53 protein has a central role in cell survival. Therefore the network that controls its concentration is rich and complex, so that modelling its dynamics 
is a challenge to mathematicians. This protein regulates essential cellular pathways, such as the ones controlling cell cycle arrest, DNA repair and apoptosis, and thereby it has a decision-making role for the cell, choosing between death and survival, and such a 'decision' must be strictly regulated by specialised cell signalling pathways. In healthy cells, p53 concentration is kept low by the action of several proteins. However after the DNA has been damaged, p53 undergoes many post-translational modifications and accumulates in the nucleus. The major p53 negative regulator, Mdm2, inhibits p53 accumulation in two principal ways: by enhancing p53 degradation by acting as an enzyme (ubiquitin ligase) and by marking p53 for degradation [3]; it also reduces p53 nuclear import by masking its principal NLS (Nuclear Localisation Signal) [4]. Furthermore, Mdm2 interacts with p53 to inhibit its transcriptional activity [5, 6]. On the other hand, p53 activates Mdm2 transcription [7] so that Mdm2 and p53 together form a negative feedback loop.

The p53 network can be activated by several independent pathways. Here we focus on the case of DNA damage (such as caused by ionising radiation or cytotoxic drug insults), where the activation of the network depends on two protein kinases: ATM and Chk2 [8, 9]. In particular, ATM responds to damaging agents that produce double strand breaks in DNA [10] and mediates phosphorylation events linked to DNA double strand breaks [11]. Finally ATM phosphorylates p53 at multiple sites [12]. In response to these stimuli, within a few minutes, the protein accumulates in the cell nucleus [13]. The half-life of p53 rises significantly because of post-translational modifications, from twenty minutes [14], when for instance no damage is sensed, to several hours, after detection of a cellular stress [15]. Several works demonstrate that p53 stabilisation is a consequence of Mdm2-p53 blocked interaction. Indeed at least 11 post-translational modifications of p53 have been reported in response to DNA damage $[8,16]$. On the one hand these modifications allow the stabilisation of p53 in the nucleus, on the other hand they regulate p53 transcriptional activity [16]. The activation of p53 is not fully controlled by any single phosphorylation site or protein [9] and the mechanisms that control p53 nuclear accumulation have not yet been completely elucidated. See for example [17] for a review on p53 and Mdm2 cellular location.

\subsection{Modelling background: mathematical models of p53 dynamics}

Within the regulatory circuit of p53, the existence of a negative feedback between p53 and Mdm2 has been clearly established [18]. The protein Mdm2 is the major p53 antagonist: as mentioned above, it enhances p53 degradation [19], blocks its entry to the nucleus [4] and inhibits p53 transcriptional activity [6]. Conversely, p53 activates Mdm2 transcription [7]. It has been shown that, in a model design perspective, a negative feedback is necessary to obtain oscillations, but it is not sufficient by itself [20]. However, the addition of a third variable to 
the system produces oscillations, at least damped ones, as shown in [1]. This third variable can represent the mRNA of one of the two proteins and serve the purpose of an underlying time delay. Sustained oscillations, as they have been observed in biological experiments, can be obtained by using an explicit time delay, namely by expliciting the dependence of protein production at time $t$ upon its concentration at $t-\tau$ for a fixed delay $\tau$, as proposed by Monk and Ma [21, 22]. Interesting though these models may be, they are not flawless with respect to physiological realism: explicit delays are meant to globally account for translation and translocation processes, and an implicit delay, i.e. a third variable, stands for an abstract putative intermediate, that lacks physiologically based definition. An oscillatory behaviour can also be reproduced by adding to the p53-Mdm2 negative loop a positive feedback, as proposed in [23, 24]. Biological evidence shows that p53 interferes with Mdm2 nuclear entry by inhibiting Mdm2 phosphorylation, which is needed for its nuclear shuttling [25]. This negative control over Mdm2 is mediated by several proteins, whose regulation is controlled by p53, but it does not depend directly on p53.

In another class of models, among which we will set the present work, the introduction of a spatial component, as already pointed out in [26, 27], allows to find the oscillations with a system of simple biochemical reactions. In a first article Sturrock et al. [27], replaced the explicit delay in the model proposed by Monk in [21] by the introduction of a spatial variable and showed that oscillations can be achieved by considering the location of biological processes. More recently the same authors, in [28], further detailed their spatial model, including both a nuclear membrane, as already done in [29,30], and the structure of cytosolic microtubules. The result of this work is a robust oscillator. The authors were indeed able to enlarge the range of parameters over which they obtain oscillations. However the values of physical parameters, such as diffusion or permeability, over which the oscillatory behaviour is observed, correspond to those of macro-molecules whose molecular weight is about $500 \mathrm{kDa}$ [31], whereas p53 and Mdm2 molecular weights are in fact about $50 \mathrm{kDa}$. It is also important to note that p53 exists as monomers, dimers and tetramers [32] and this takes its molecular weight to higher values.

In the present work, we do not consider a complicated biological network in order to introduce a positive feedback. Neither do we use delays that would leave model details hidden to the understanding of the whole mechanism and would hinder its future physiological identification. We develop a model that takes into account the intracellular spatial structure of the cell in the reconstruction of the dynamics of p53. This allows us to base our model only on well-established biological hypotheses and to use realistic biophysical coefficients, related to molecule diffusion or nuclear membrane permeability. In particular, we consider, unlike [28], that transport of particles through the membrane can be unidirectional, as biology 
teaches us. In this way we also emphasize the mechanisms of activation of p53 linked to enhanced nuclear import and reduced nuclear export. Furthermore, the observed 'delays' due to transport or transcription can be explained using diffusion or translocation, and molecular reactions can be physiologically located. We obtain a model in which the behaviour of cell signalling mechanisms is strictly related to the basic spatial distinction between nucleus and cytoplasm.

\section{An ODE compartmental model}

In this section we introduce the model we intend to analyse in this work. We present it as a compartmental ODE system and we still do not consider the spatial component, by assuming that the concentrations of proteins are homogeneous in each compartment. The model involves two distinct states of p53, its primary inhibitor Mdm2, and the mRNA of Mdm2 whose synthesis is promoted by p53. Every species of the model exists in a nuclear and in a cytoplasmic form. If necessary, to avoid confusion between the two compartments, we denote by $[\cdot]^{(n)}$ and $[\cdot]^{(c)}$, respectively, the nuclear and cytoplasmic concentration of each species. Protein p53 undergoes several conformational changes. In our model we represent the ubiquitination process, i.e. the enzymatic action of Mdm2 on p53 by which p53 is marked for degradation. Even if ubiquitination is more complicated than other enzymatic processes, we choose, for the sake of simplicity, to model it as a classical enzymatic reaction. Thus, knowing the interactions of p53 and Mdm2, we can write the following:

$$
[M d m 2]+[p 53] \underset{k_{-}}{\stackrel{k}{\rightleftharpoons}}[\text { Complex }] \stackrel{k_{3}}{\rightarrow}\left[p 53_{U}\right]
$$

where $p 53_{U}$ represents the ubiquitinated form of $\mathrm{p} 53$. Then, following the Law of Mass Action and applying the Quasi Steady State Approximation [33, 34], the associated differential equation describing the chemical kinetics in (1) is given by:

$$
\frac{d[p 53]}{d t}=-k_{2}[M d m 2] \frac{[p 53]}{K_{1}+[p 53]},
$$

where $K_{1}=\frac{k_{1}+k_{-}}{k}$, and $k_{2}[M d m 2]$ represents the maximum rate of the reaction. The right term of equation (2) represents a loss of mass in our system since we suppose that ubiquitinated p53 will be degraded by the proteasome. In order to be detected by the proteasome, p53 actually needs to be ubiquitinated several times (see [5] and reference therein for a review on Mdm2-mediated p53 ubiquitination). We choose not to describe all the single ubiquitination steps, but rather a global one, because we are not interested in describing how p53 exits the system, but 
how it is activated. We also take into account the phosphorylation and dephosphorylation process of p53. As explained in Section 2.1, a single conformational change cannot uniquely determine the activity of the p53 protein. Several posttranslational transformations decide how p53 will be activated [16] and stabilised. Since p53 phosphorylation is particularly important for its regulation in response to DNA damage [35], we represent only this transformation and we treat the phosphorylated form of p53 as a generic 'active' species. Furthermore, although we are aware of the fact that different kinases are activated and are able to phosphorylate p53 depending on the type of damage, we choose to model here only the ATM kinase, that is thus here meant to roughly represent a DNA damage sensor. For the sake of simplicity we treat ATM as parameter and consider simple enzymatic kinetics. We consider the following reactions:

$$
\begin{gathered}
{[\text { ATM }]+[p 53] \underset{k_{-2}}{\stackrel{k_{2}}{\rightleftharpoons}}[\text { Complex }] \stackrel{k_{3}}{\rightarrow}\left[p 53_{p}\right],} \\
{[E]+\left[p 53_{p}\right] \underset{k_{-4}}{\stackrel{k_{4}}{\rightleftharpoons}}[\text { Complex }] \stackrel{k_{5}}{\rightarrow}[p 53],}
\end{gathered}
$$

where $p 53_{p}$ is the phosphorylated form of $\mathrm{p} 53$, while $[E]$ is a generic phosphatase that removes a phosphate group from its substrate $p 53_{p}$. Again by the Law of Mass Action and the quasi Steady State Approximation we obtain the following equations:

$$
\begin{aligned}
& \frac{d[p 53]}{d t}=k_{p h} \frac{\left[p 53_{p}\right]}{K_{p h}+\left[p 53_{p}\right]}-k_{3}[A T M] \frac{[p 53]}{K_{A T M}+[p 53]} \\
& \frac{d\left[p 53_{p}\right]}{d t}=k_{3}[A T M] \frac{[p 53]}{K_{A T M}+[p 53]}-k_{p h} \frac{\left[p 53_{p}\right]}{K_{p h}+\left[p 53_{p}\right]},
\end{aligned}
$$

where $k_{p h}$ is the maximum velocity of the dephosphorylation process, $K_{p h}=\frac{k_{5}+k_{-4}}{k_{4}}$ and $K_{a t m}=\frac{k_{3}+k_{-2}}{k_{2}}$. We assume that the phosphorylated form of $\mathrm{p} 53$, that is the product of the reaction in equation (3), is unable to interact with Mdm2, so that after DNA damage, a pool of p53 is not subject to Mdm2 control and is active in the nucleus as a transcription factor. We then model protein transcription and translation: the mRNA of Mdm2 is produced in the nucleus at a constant rate but also in a p53-dependent manner. Since p53 is known to be active as a transcription factor when it is formed out of four sub-units, we represent, as is classical in modelling cooperative processes [36], this reaction by using a Hill function with coefficient 4 :

$$
\frac{d\left[M d m 2_{R N A}\right]}{d t}=k_{S m}+k_{S p} \frac{\left(\left[p 53_{p}\right]\right)^{4}}{\left(\left[p 53_{p}\right]\right)^{4}+K_{S p}^{4}},
$$


these reactions being located only in the nucleus. The mRNA of Mdm2 is then translated in the cytoplasm by the ribosomes, located only in the cytoplasm. We assume a constant production rate for the synthesis of p53 in the cytoplasmic compartment. Indeed, we do not have any biological evidence about changes of p53 transcription after a cell damage and therefore we choose not to represent explicitly its mRNA. Based on our assumptions we obtain the following differential equation system for the activity of the coupled oscillator p53-Mdm2 in the nucleus:

$$
\left\{\begin{array}{l}
\frac{d[p 53]^{(n)}}{d t}=k_{p h} \frac{\left[p 53_{p}\right]^{(n)}}{K_{p h}+\left[p 53_{p}\right]^{(n)}}-k_{1}[M d m 2]^{(n)} \frac{[p 53]^{(n)}}{K_{1}+[p 53]^{(n)}}-\frac{k_{3}[A T M][p 53]^{(n)}}{K_{A T M}+[p 53]^{(n)}} \\
-\rho_{p} V_{r}\left([p 53]^{(n)}-[p 53]^{(c)}\right), \\
\frac{d[M d m 2]^{(n)}}{d t}=-\delta_{m}[M d m 2]^{(n)}-\rho_{m} V_{r}\left([M d m 2]^{(n)}-[M d m 2]^{(c)}\right), \\
\frac{d\left[M d m 2_{R N A}\right]^{(n)}}{d t}=k_{S m}+k_{S p} \frac{\left(\left[p 53_{p}\right]^{(n)}\right)^{h}}{\left(\left[p 53_{p}\right]^{(n)}\right)^{h}+K_{S p}^{h}}-\delta_{r}\left[M d m 2_{R N A}\right]^{(n)} \\
\frac{d\left[p 53_{p}\right]^{(n)}}{d t}=\rho_{q} V_{r}\left[p 53_{p}\right]^{(c)}+\frac{k_{3}[A T M][p 53]^{(n)}}{K_{A T M}+[p 53]^{(n)}}-k_{p h} \frac{\left[p 53_{p}\right]_{r}^{(n)} V_{r}\left[M d m 2_{R N A}\right]^{(n)}}{K_{p h}+\left[p 53_{p}\right]^{(n)}},
\end{array}\right.
$$

and the following one in the cytoplasm:

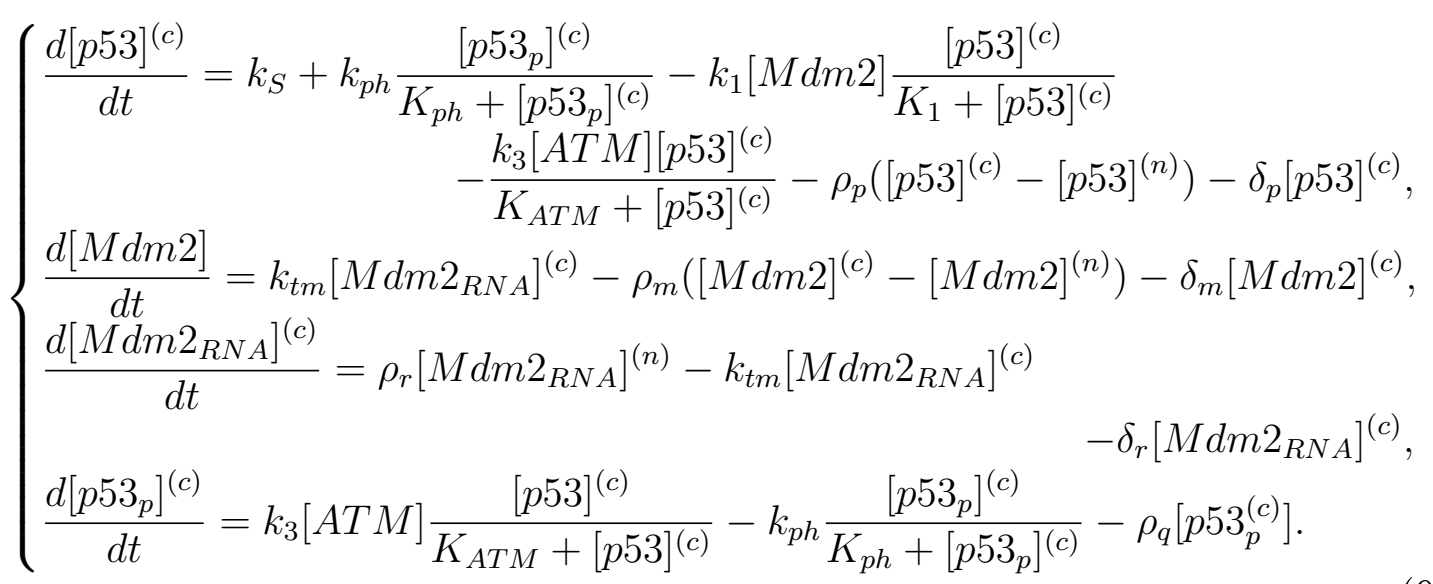

Note that we denoted by $h$ the exponent of the Hill term in the equation for $\left[M d m 2_{R N A}\right]^{(n)}$. Here we include degradation terms as linear functions of each protein concentration. Since it has been proved that p53 degradation occurs mainly in the cytoplasm [4], we add, besides the ubiquitination term, a classical degradation term for p53, in the cytoplasm. Nuclear p53 also undergoes degradation $([37,38])$, but in normal growth conditions this is not the preferential way chosen by the cell [19]. Phosphorylated p53 poorly interacts with Mdm2 and it is thus not marked for degradation by Mdm2. We assume that it will not be degraded at 
all and do not consider any degradation term. Following Ciliberto et al. in [24], we model the exchanges between compartments as a linear contribution of a difference between averaged nuclear and cytoplasmic concentrations and we multiply the nuclear flux by $V_{r}$, a nondimensional quantity representing the volume ratio between cytoplasm and nucleus. This choice will be also supported by further discussion in Section 4. It is known that p53 and Mdm2 can shuttle between nucleus and cytoplasm $[4,7]$. On the contrary nuclear export of phosphorylated p53, that represents active p53, is inhibited after DNA damage [39, 40]. Therefore, basing our modelling hypotheses on these biological observations, we assume here that p53 and Mdm2 can traverse the different compartments. The phosphorylated form of p53 is assumed to move from cytoplasm to nucleus but not backwards. On the contrary, we assume that the mRNA of Mdm2 only moves from the nucleus, where it is transcribed, to the cytoplasm, where it is translated.

\subsection{Sustained Oscillations appear in the ODE model.}

In this section we begin the study of our model in the compartmental setting in order to capture the temporal dynamics of the system [41]. To simplify our notations, let us set $p=[p 53], m=[M d m 2], r=\left[M d m 2_{R N A}\right]$ and $q=\left[p 53_{P}\right]$. In the sequel we denote the nuclear and cytoplasmic concentrations by the superscripts $(n)$ and $(c)$. Finally the autonomous differential system resulting from the model presented in Section 3 may be written as:

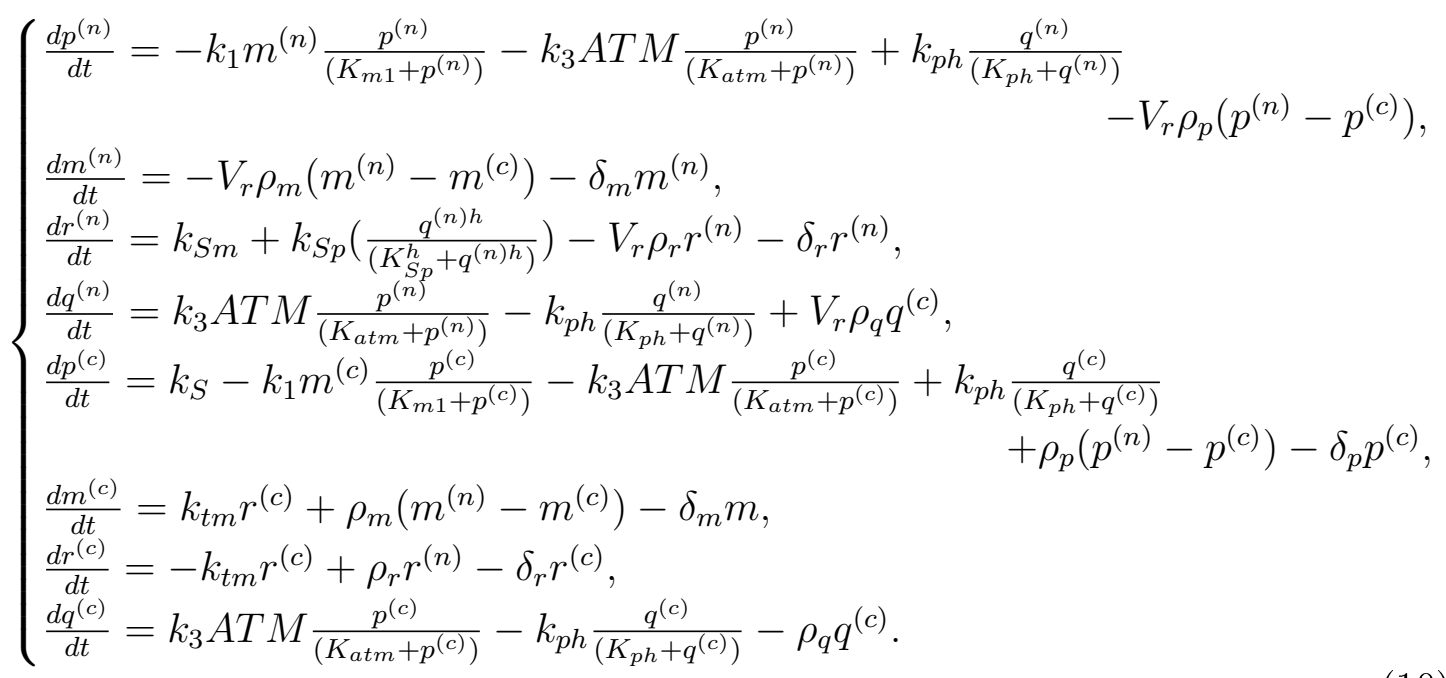

Here the first four equations represent the nuclear concentrations and the others the cytoplasmic ones.

First of all we nondimensionalise the system by writing

$$
\begin{gathered}
\bar{p}(\tau)=\frac{p(t)}{\alpha_{p}}, \ldots, \bar{q}(\tau)=\frac{q(t)}{\alpha_{q}} \\
\tau=\frac{t}{t^{\star}}
\end{gathered}
$$


where the $\alpha_{s}$ are concentrations of the ' $s$ ' species, $s=p, m, r, q$ (expressed in $\left.\mu M\right)$, and $t^{\star}$ is a time constant, expressed in minutes. We fixed $\alpha_{p}=\alpha_{q}$ as p53 reference concentrations and $\alpha_{m}=\alpha_{r}$ as Mdm2 reference concentrations. Then we chose $\alpha_{p}=K_{a t m}, t^{*}=\frac{1}{k_{3}}$ and $\alpha_{m}=\frac{k_{S p}}{k_{3}}$. Thus the nondimensional system depends on less parameters than the dimensional one. Namely it does not depend on $K_{a t m}, k_{3}$ and $k_{S p}$, while retaining all of its geometry. The nondimensional can be written as follows:

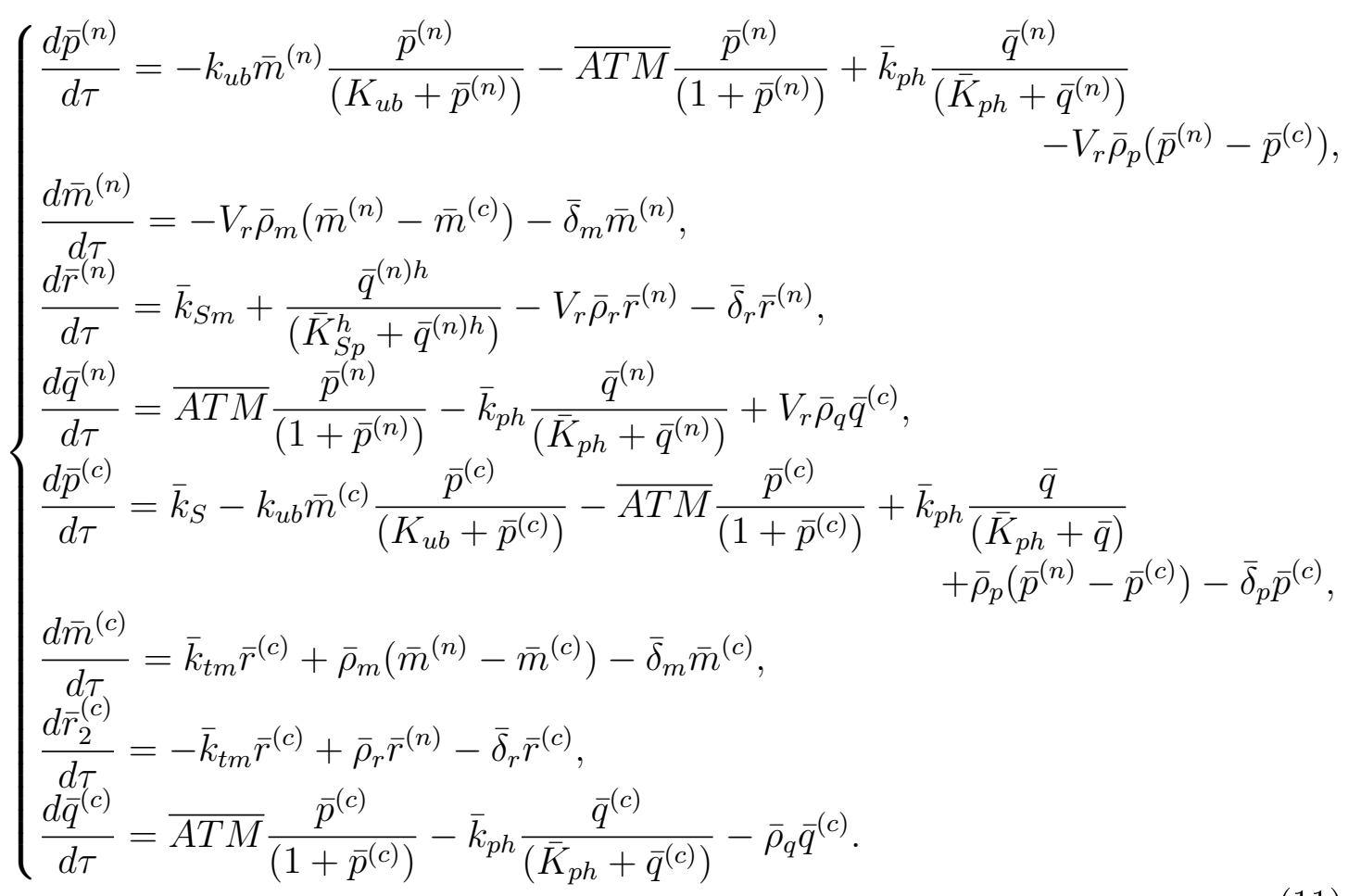

Here we set: $k_{u b}:=t^{\star} k_{4} \frac{\alpha_{m}}{\alpha_{p}}, K_{u b}:=\frac{K_{m 1}}{\alpha_{p}}, \overline{A T M}:=\frac{A T M}{\alpha_{p}}, \bar{k}_{p h}:=\frac{k_{p h} t^{\star}}{\alpha_{p}}, \bar{K}_{p h}:=\frac{K_{p h}}{\alpha_{p}}$, $\bar{k}_{S m}=\frac{t^{\star} k_{S m}}{\alpha_{m}}, \bar{K}_{S p}:=\frac{K_{S p}}{\alpha_{p}}, \bar{k}_{t p}:=\frac{t^{\star} k_{t p}}{\alpha_{0}}, \bar{k}_{S}:=t^{\star} k_{S}$. Then $\bar{\rho}_{s}=t^{\star} \rho_{s}$ and $\bar{\delta}_{s}=t^{\star} \delta_{s}$ for $s=p, m, r, q$. We performed simulations of this system, using the values of the parameters on column 3 of Table 1. Those values have been obtained by numerical data fitting, starting from values found in the biological or modelling literature on p53. Next we found for each parameter a range of values for which the oscillations are present. To do this, we fixed all the parameters, except for one that we varied, to assess the response of the system. These ranges correspond in order of magnitude to the corresponding parameters of many other models [24, 42, 43].

In Figure 1(a) we represent the temporal evolution of nuclear concentrations of phosphorylated p53 and Mdm2 (respectively $\bar{q}^{(n)}$ and $\bar{m}^{(n)}$ ). We can observe sustained oscillations of their concentrations. On 1(b) we show the phase plane relative to the same simulation where we can observe the corresponding limit cycle 
in the $\left(\bar{q}^{(n)}, \bar{m}^{(n)}\right)$ plane (respectively nuclear $p 53_{p}$ and $M d m 2$ ).

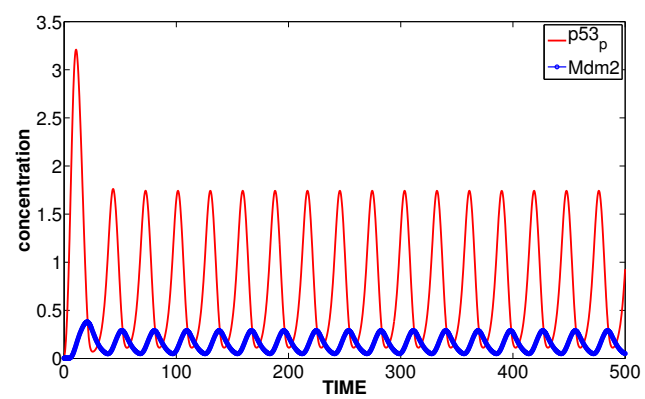

(a)

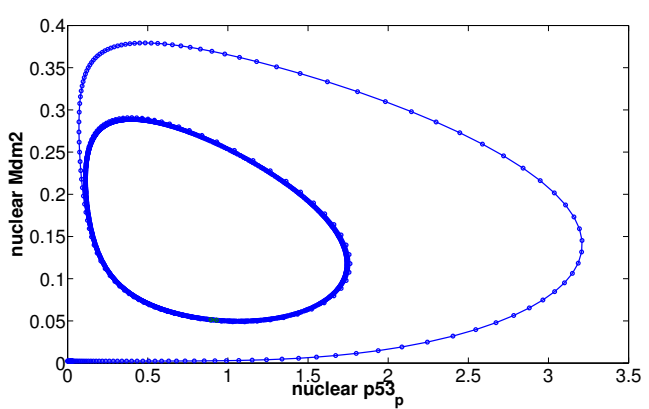

(b)

Figure 1: ODE Model. Simulations results. (a): Evolution of nuclear concentrations of $M d m 2$ and $p 53_{p}$ for $\overline{A T M}=30$, a value in the range [1.4, 97.5], giving rise to sustained oscillations (see section 3.1.1) . (b): Phase plane relative to the variable $\bar{m}^{(n)}$ and $\bar{q}^{(n)}$ (nuclear $M d m 2$ and nuclear $p 53_{p}$ ). The orbit of the solution tends towards a stable limit cycle.

\subsubsection{A supercritical Hopf Bifurcation explains the oscillations}

In healthy cells, p53 is targeted by Mdm2-mediated ubiquitination and it is highly degraded by the cell machinery. The damage sensor ATM is inactive and no oscillations are present, which our model reproduces accurately, as shown below. On the contrary, when a damage to the DNA occurs, conformational transformations of both Mdm2 and p53 allow p53 to be released from tight control, allowing a pool of active p53 to accumulate and oscillations to begin. In system (11) we set $\bar{q}=p 53_{p}$ as the free and active species and the growth of its concentration is related to the phosphorylation process, exerted by ATM. Thus, if $\overline{A T M}=0$, i.e. if ATM is inactive, $\bar{q}$ tends to zero and no oscillations appear, as is easily seen in system (11). Using the numerical values given in Table 1 , it is possible to numerically determine the coordinates of the equilibrium point for $\overline{A T M}=0$. Setting $\bar{c}=\left[\bar{p}^{(n)}, \bar{m}^{(n)}, \bar{r}^{(n)}, \bar{q}^{(n)}, \bar{p}^{(c)}, \bar{m}^{(c)}, \bar{r}^{(c)}, \bar{q}^{(c)}\right]$ we calculate the stable equilibrium point whose coordinates are: $\bar{c}=[0.2364,0.0024,0.0125,0,0.3028,0.0029,0.0005,0]$. Note that in this set of values determined for $\overline{A T M}=0$, the coordinates of the equilibrium point, owing to the transcription source terms, are nonnegative, except for the actual activity variables $p 53_{p}$ (nuclear and cytoplasmic), that are triggered from zero only if $\overline{A T M}>0$. Then, by a classical continuation-bifurcation method, we determined the equilibrium point for each positive value of $\overline{A T M}$. When we increase the value of the parameter $\overline{A T M}$ the equilibrium value of $\bar{q}$ becomes strictly positive in the cytoplasm and in the nucleus. As a consequence, the p53-Mdm2 negative feedback turns on and for values of $\overline{A T M}$ high enough we reproduce the well-known oscillations of the p53 system. We find that for values of $\overline{A T M}<1.4$ 


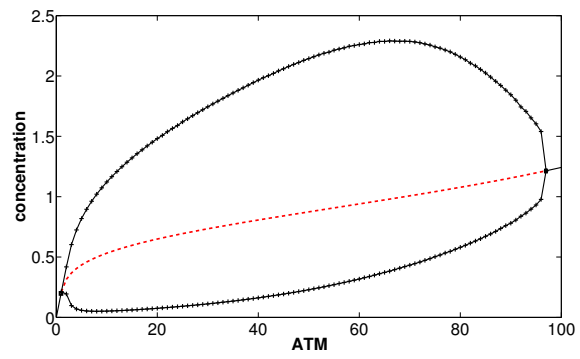

(a) Bifurcation diagram of $\bar{q}^{(n)}$

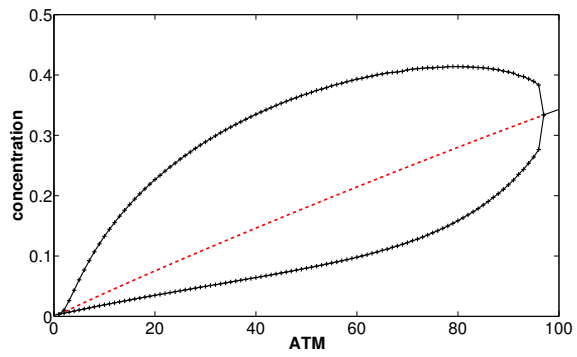

(b) Bifurcation diagram of $\bar{m}^{(n)}$

Figure 2: Bifurcation diagrams (ODE system) for nuclear $p 53_{p}$ and nuclear $M d m 2$. The equilibrium of the system is stable for values of $\overline{A T M}$ lower than a threshold $(\overline{A T M} \sim 1.4)$, then a supercritical Hopf bifurcations occurring, it becomes unstable and a limit cycle appears. The dotted curve represents the branch with unstable equilibrium and the + marked curves represent the minimum and maximum values of the oscillations. When $\overline{A T M}$ becomes higher then at a second threshold the equilibrium becomes stable again through a second supercritical Hopf bifurcation $(\overline{A T M} \sim 97.5)$.

and $\overline{A T M}>97.5$, the corresponding equilibrium points of system (11) are stable since all the eigenvalues of the linearized system are strictly negative [44]. At $\overline{A T M} \sim 1.4$ and $\overline{A T M} \sim 97.5$ two supercritical Hopf bifurcations occur. Indeed, all the hypotheses necessary to apply the Hopf Theorem are satisfied [45] (which we did verify by numerical simulations, data not shown) and a supercritical Hopf bifurcation actually occurs. More technical details about the bifurcation analysis of this ODE system, will be given in a future work. For all values of $\overline{A T M} \in[1.4,97.5]$, the equilibrium point of system (11) is unstable and a stable limit cycle exist, towards which the solution of the system tends. Thus the system oscillates and reproduces the observed biological behaviour. As proposed in [22], our results can be interpreted as follows. If the damage is too low, the cell does not need to activate the p53 pathway in order to repair. When the damage is high enough, the p53 pathway needs to be launched and the oscillations begin. On the contrary, if the damage is too big, the cell does not start repair processes, but directly launches apoptosis. Figure 2 presents the bifurcation diagram of $\bar{q}^{(n)}$ and $\bar{m}^{(n)}$. The dotted curves represent the value of the unstable equilibrium for each of the variables. The +-marked curves represent the oscillation amplitude that, as we can observe, varies slightly. The period of oscillations varies between 25 and 33 min for values of $\overline{A T M} \in[1.4,97.5]$ (results not shown). This observation is consistent with biological experiments showing that the amplitude of oscillations varies with growing damage level, while the period is more stable when the damage level varies [46]. The characteristic period of these numerical simulations is shorter than the experimental one $(\sim 4-5 h[46])$. However, the exact values of the parameters in Table 1 are not easy to be determined physiologically, and 


\begin{tabular}{|c|c|c|c|c|}
\hline Param. & Description & Chosen Value & Units & Ranges for osc. \\
\hline$k_{p h}$ & Dephosphorylation rate & 0.1 & $\mu M / \min$ & $0.039 \leq k_{p h} \leq 1.73$ \\
\hline$K_{p h}$ & Michaelis dephosporylation constant & 0.05 & $\mu M$ & $0.0001 \leq K_{p h} \leq 2.2$ \\
\hline$k_{1}$ & Ubiquitination rate & 100 & $\min ^{-1}$ & $30.5 \leq k_{1} \leq 4180$ \\
\hline$K_{1}$ & Michaelis ubiquination constant & 1.01 & $\mu M$ & $0.023 \leq K_{1} \leq 3.3$ \\
\hline$k_{3}$ & Phosphorylation velocity & 1 & $\min ^{-1}$ & fixed \\
\hline$K_{a t m}$ & Michaelis phosphorylation constant & 0.1 & $\mu M$ & fixed \\
\hline$\delta_{m}$ & Mdm2 degradation rate & 0.16 & $\min ^{-1}$ & $0.03 \leq \delta_{m} \leq 0.45$ \\
\hline$k_{S m}$ & basal Mdm2 mRNA transcription rate & 0.005 & $\mu M / \min$ & $0 \leq k_{S m} \leq 0.19$ \\
\hline$k_{S p}$ & p53-dependent Mdm2 mRNA transcription rate & 1 & $\min ^{-1}$ & fixed \\
\hline$K_{S p}$ & Michaelis p53-dependent Mdm2 mRNA transcription & 0.1 & $\mu M$ & $0.06 \leq K_{S p} \leq 0.9$ \\
\hline$h$ & Hill coefficient & 4 & adim & $h \geq 2$ \\
\hline$\delta_{r}$ & Mdm2 mRNA degradation rate & 0.0001 & $\min ^{-1}$ & $0 \leq \delta_{m R N A} \leq 0.41$ \\
\hline$k_{S}$ & p53 synthesis rate & 0.015 & $\mu M / \min$ & $0.001 \leq k_{S} \leq 0.02$ \\
\hline$\delta_{p}$ & p53 degradation rate & 0.2 & $\min ^{-1}$ & $0 \leq \delta_{p 53} \leq 23$ \\
\hline$k_{t m}$ & Mdm2 translation rate & 1 & $\min ^{-1}$ & $\forall k_{t m} \geq 0.02$ \\
\hline$V_{r}$ & Volume ratio & 10 & adim & $0.8 \leq V_{r} \leq 24.2$ \\
\hline$\rho_{p}$ & $p 53$ permeability & 0.083 & $\min ^{-1}$ & $\rho_{p} \geq 0$ \\
\hline$\rho_{m}$ & Mdm2 permeability & 0.04 & $\min ^{-1}$ & $\rho_{m}>0$ \\
\hline$\rho_{r}$ & Mdm2 mRNA permeability & 0.083 & $\min ^{-1}$ & $\rho_{r}>0$ \\
\hline$\rho_{q}$ & $p 53_{p}$ permeability & 0.083 & $\min ^{-1}$ & $\rho_{q}>0.01$ \\
\hline
\end{tabular}

Table 1: Parameter values for system (10). Starting from values taken from [42, 43] and [24] we numerically obtained by data fitting, a complete set of parameter values that are used in the sequel for the simulations. Keeping all but one of these parameters fixed at these reference values, and varying the last one, we also calculated ranges of parameters (in projection on each parameter axis) for which sustained oscillations take place. Note that the parameters $k_{3}, K_{a t m}$ and $k_{S p}$ are presented in this table as fixed because they have been fixed to constant values by the change of variables occurring in the nondimensionalisation process (see text in Section 3.1).

we will perform such physiological interpretation only in the PDE case with the introduction of space. We will indeed show in the next Sections how we can obtain more realistic periods in our model. From this preliminary study of the ODE compartmental model, we verified, in a simplified setting, that the system proposed reproduces the expected behaviour, with no need of any artificial mechanism. In the next sections we analyse the behaviour of the system in a spatial setting and we study its stability under spatial perturbations.

\section{Adding the spatial variable: the PDE case}

In this section we complete the setting of our model, introducing a spatial variable. 


\subsection{Mathematical formulation of the model in the 2-dimensional case}

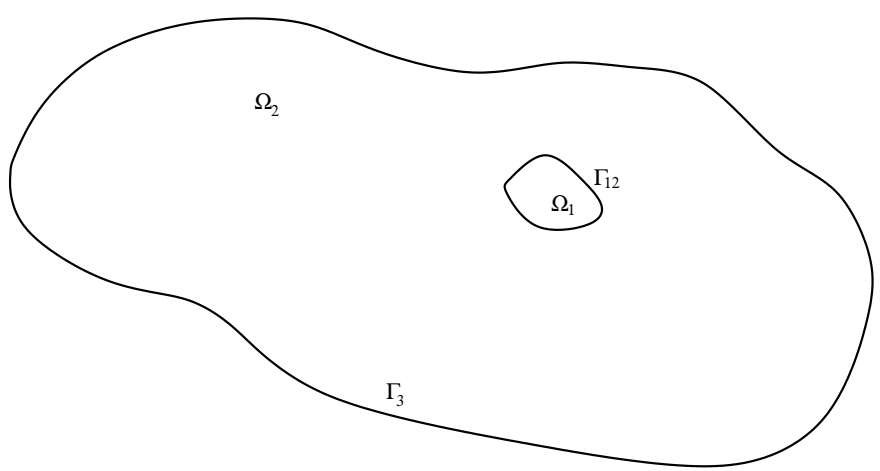

Figure 3: Domain of the PDE system: we represent the two different compartments, $\Omega_{1}$ for the nucleus and $\Omega_{2}$ for the cytoplasm. $\Gamma_{12}$ is the common boundary between the two compartments; $\Gamma_{3}$ is the exterior boundary, representing the cellular membrane.

We cast our system in a 2-dimensional domain divided into a nuclear and a cytoplasmic compartment as shown in Figure 3. We set $\Omega$ as the entire domain of the cell and we define $\Omega_{1}$ as the nuclear compartment and $\Omega_{2}$ as the cytoplasmic one. We set $\Gamma_{12}:=\partial \Omega_{1} \cap \partial \Omega_{2}$ for the common boundary and $\Gamma_{3}:=\partial \Omega \backslash \Gamma_{12}$. Each species is a function of time $t$ and position $\mathbf{x}=(x, y)$; we suppose that each species is able to diffuse in the cytoplasm and in the nucleus. We include permeability conditions at the common boundary $\Gamma_{12}$ that take into account the nuclear membrane. As for the ODE case, we nondimensionalise the system. Setting

$$
\begin{aligned}
\bar{p}(\xi, \eta, \tau) & =\frac{p(x, y, t)}{\alpha_{p}}, \ldots, \bar{q}(\tau)=\frac{q(x, y, t)}{\alpha_{q}}, \\
\tau & =\frac{t}{t^{\star}}, \xi=\frac{x}{L}, \eta=\frac{y}{L},
\end{aligned}
$$

where $L$, expressed in $\mu m$, is some 1-dimensional space parameter related to cell size, we obtain in $\Omega_{1}$ :

$$
\left\{\begin{array}{l}
\frac{\partial \bar{p}}{\partial \tau}=\bar{D}_{p} \Delta \bar{p}-k_{u b} \bar{m} \frac{\bar{p}}{\left(K_{u b}+\bar{p}\right)}-\overline{A T M} \frac{\bar{p}}{(1+\bar{p})}+\bar{k}_{p h} \frac{\bar{q}}{\left(K_{p h}+\bar{q}\right)} \\
\frac{\partial \bar{m}}{\partial \tau}=\bar{D}_{m} \Delta \bar{m}-\bar{\delta}_{m} \bar{m} \\
\frac{\partial \bar{r}}{\partial \tau}=\bar{D}_{r} \Delta \bar{r}+\bar{k}_{S m}+\frac{\bar{q}^{h}}{\left(\bar{K}_{S p}^{h}+\bar{q}^{h}\right)}-\bar{\delta}_{r} \bar{r} \\
\frac{\partial \bar{q}}{\partial \tau}=\bar{D}_{q} \Delta \bar{q}+\overline{A T M} \frac{\bar{p}}{(1+\bar{p})}-\bar{k}_{p h} \frac{\bar{q}}{\left(\bar{K}_{p h}+\bar{q}\right)}
\end{array}\right.
$$

and in $\Omega_{2}$ :

$$
\left\{\begin{array}{l}
\frac{\partial \bar{p}}{\partial \tau}=\bar{D}_{p} \Delta \bar{p}+\bar{k}_{S}-k_{u b} \bar{m} \frac{\bar{p}}{\left(K_{u b}+\bar{p}\right)}-\overline{A T M} \frac{\bar{p}}{(1+\bar{p})}+\bar{k}_{p h} \frac{\bar{q}}{\left(\bar{K}_{p h}+\bar{q}\right)}-\bar{\delta}_{p} \bar{p}, \\
\frac{\partial \bar{m}}{\partial \tau}=\bar{D}_{m} \Delta \bar{m}+\bar{k}_{t m} \bar{r}-\bar{\delta}_{m} \bar{m}, \\
\frac{\partial \bar{r}}{\partial \tau}=\bar{D}_{r} \Delta \bar{r}-\bar{k}_{t m} \bar{r}-\bar{\delta}_{r} \bar{r} \\
\frac{\partial \bar{q}}{\partial \tau}=\bar{D}_{q} \Delta \bar{q}+\overline{A T M} \frac{\bar{p}}{(1+\bar{p})}-\bar{k}_{p h} \frac{\bar{q}}{\left(\bar{K}_{p h}+\bar{q}\right)},
\end{array}\right.
$$


where we used the same notations as in section 3.1. Notice that, as in [29], we used Fick's Law to model the diffusion of each species concentration. We defined $\bar{D}_{s}=$ $\frac{t^{\star} D_{s}}{L^{2}}, i=0, \ldots, 3$, as the nondimensional diffusion coefficients, where $D_{s}$ is the dimensional diffusion parameter of the ' $s$ ' species, expressed in $\mu \mathrm{m}^{2} / \mathrm{min}$. Finally, following [29] and [30], and consistently with the assumptions of the ODE model, we fix Kedem-Katchalsky [47] boundary conditions at the common boundary $\Gamma_{12}$ for the species that cross the nuclear membrane from both sides:

$$
\begin{cases}\frac{\partial \bar{p}^{n}}{\partial \mathbf{n}}=\frac{\bar{\rho}_{p}}{\bar{D}_{p}}\left(\bar{p}^{c}-\bar{p}^{n}\right)=\frac{\partial \bar{p}^{c}}{\partial \mathbf{n}} & \text { on } \Gamma_{12}, \\ \frac{\partial \bar{m}^{n}}{\partial \mathbf{n}}=\frac{\bar{\rho}_{m}}{\bar{D}_{m}}\left(\bar{m}^{c}-\bar{m}^{n}\right)=\frac{\partial \bar{m}^{c}}{\partial n} & \text { on } \Gamma_{12}\end{cases}
$$

meaning that the flux of each species through the nuclear envelope is proportional to the difference between the concentrations at the two sides of the membrane. Notice that the normal vector $\mathbf{n}$ is pointing outwards from the nucleus and that fluxes are continuous. We fix the following boundary conditions for $\bar{r}$ and $\bar{q}$ :

$$
\begin{cases}\frac{\partial \bar{r}^{n}}{\partial \mathbf{n}}=\frac{\bar{\rho}_{r}}{\bar{D}_{r}}\left(-\bar{r}^{n}\right)=\frac{\partial \bar{r}^{c}}{\partial \mathbf{n}} & \text { on } \Gamma_{12}, \\ \frac{\partial \bar{q}^{n}}{\partial \mathbf{n}}=\frac{\bar{\rho}_{q}}{\bar{D}_{q}} \bar{q}^{c}=\frac{\partial \bar{q}^{c}}{\partial \mathbf{n}} & \text { on } \Gamma_{12},\end{cases}
$$

that include the directionality of transport: $\bar{r}$, the mRNA of Mdm2, is only able to exit the nucleus, while $\bar{q}$, phosphorylated p53, traverses the nuclear membrane only towards the nucleus. Again notice that the fluxes are continuous. The constants $\bar{\rho}_{s}$ are the permeability coefficients relative to each species and are expressed, in the dimensional model, in $\mu \mathrm{m} / \mathrm{min}$. We also assume that proteins are not able to exit the cell and we use Neumann homogeneous boundary conditions on $\Gamma_{3}$ (zero-flux) for all species:

$$
\frac{\partial s}{\partial \mathbf{n}}=0 \text { on } \Gamma_{3}, \quad s=p, m, r, q .
$$

Since nuclear pores are homogeneously distributed on the nuclear envelope and far from saturation [30], the choice of Kedem-Katchalsky boundary conditions, to model membrane permeability [47], is consistent with our environment; see [29] and references therein for more details on Kedem-Katchalsky conditions in the case of the nuclear membrane. We emphasize here that the choice of the boundary condition is coherent with the choice of the ODE model, where we fixed an exchange rule between compartments as the linear contribution of the difference of the mean concentrations in each compartment. Let us consider a simplified system for one generic species $s$ that can only diffuse in the domain $\Omega$ :

$$
\begin{cases}\frac{\partial s^{(n)}}{\partial t}=D_{n} \Delta s^{(n)} & \text { in } \Omega_{1} \\ \frac{\partial s^{(c)}}{\partial t}=D_{c} \Delta s^{(c)} & \text { in } \Omega_{2}\end{cases}
$$


and let us couple this system by using the boundary conditions:

$$
D_{n} \frac{\partial s^{(n)}}{\partial \mathbf{n}}=\rho\left(s^{(c)}-s^{(n)}\right)=D_{c} \frac{\partial s^{(c)}}{\partial \mathbf{n}} \quad \text { on } \Gamma_{12},
$$

closed by the continuity of the flux: $D_{n} \frac{\partial s^{(n)}}{\partial \mathbf{n}}=D_{c} \frac{\partial s^{(c)}}{\partial \mathbf{n}}$. Here again the normal vector $\mathbf{n}$ is the same on both sides. If we integrate over the whole region and apply Green's formula, we obtain:

$$
\left\{\begin{array}{l}
\frac{d s^{(n)}}{d t}=\frac{\rho}{\left|\Omega_{1}\right|} \int_{\Gamma_{12}}\left(s^{(c)}-s^{(n)}\right) d \sigma, \\
\frac{d s^{(c)}}{d t}=\frac{\rho}{\left|\Omega_{2}\right|} \int_{\Gamma_{12}}\left(s^{(n)}-s^{(c)}\right) d \sigma,
\end{array}\right.
$$

thus reducing the initial system (17) to system (19) above, in agreement with equations:

$$
\frac{d s^{(n)}}{d t}=\rho V_{r}\left(s^{(c)}-s^{(n)}\right), \quad \frac{d s^{(c)}}{d t}=\rho\left(s^{(n)}-s^{(c)}\right),
$$

which corresponds to our choices in the ODE model.

\subsection{Spatial parameters}

In this new setting, the number of parameters of the system has increased. Diffusion and permeability coefficients need to be set accordingly to the biophysical knowledge of the cellular environment and molecular species. Since, according to [29], the diffusion coefficient of a general protein with a mass of about $40 k D a$ is roughly $600 \mu \mathrm{m}^{2} / \mathrm{min}$, we take this value as a reference for all the protein species, p53 and Mdm2, in the nucleus and in the cytoplasm. Using single molecule tracking and statistical analysis, recent works managed to calculate the mRNA diffusion coefficient $[48,49,50]$ and found that the coefficient of a single mRNA particle is $\sim 6-30 \mu \mathrm{m}^{2} / \mathrm{min}$, i.e., the ratio between mRNA and protein diffusion coefficients is about $1: 100$. Concerning the permeability coefficient, we supposed, to tie in with this diffusion coefficient ratio, that the ratio between the mRNA and the protein permeability coefficient is $1: 100$. In the sequel, all the simulations will be done for values of parameters listed in Table 2.

\subsection{Simulations results in a 1-dimensional domain}

We begin the study of the system in a simplified 1-dimensional domain, plotted in Figure 4. This domain is given by two adjoining segments that represent the cytoplasmic and nuclear compartments. We fix an interval $[a, c]=[0,10]$ (in $\mu m)$, $[a, c]=[a, b] \cup[b, c]$, with $[a, b]=[0,9]$ the cytoplasmic compartment, and $[b, c]=$ $[9,10]$ the nucleus, as shown in Figure 4 . We performed all simulations using finite differences schemes and we used a basic IMEX numerical scheme. For further details see [52]. Even if the geometry is oversimplified, there is a striking difference 


\begin{tabular}{|c|c|c|c|}
\hline Parameter & Description & Value & Reference \\
\hline$D_{p}$ & p53 Diffusion coefficient & $600 \mu \mathrm{m}^{2} / \mathrm{min}$ & {$[29,51]$} \\
$D_{m}$ & Mdm2 Diffusion coefficient & $600 \mu \mathrm{m}^{2} / \mathrm{min}$ & {$[29]$} \\
$D_{r}$ & Mdm2 mRNA Diffusion coefficient & $6 \mu \mathrm{m}^{2} / \mathrm{min}$ & {$[48,49]$} \\
$D_{q}$ & $p 53_{P}$ Diffusion coefficient & $600 \mu \mathrm{m}^{2} / \mathrm{min}$ & {$[29,51]$} \\
$\rho_{p}$ & $\mathrm{p} 53$ permeability coefficient & $10 \mu \mathrm{m} / \mathrm{min}$ & obtained \\
$\rho_{m}$ & Mdm2 permeability coefficient & $10 \mu \mathrm{m} / \mathrm{min}$ & obtained \\
$\rho_{r}$ & Mdm2 mRNA permeability coefficient & $0.1 \mu \mathrm{m} / \mathrm{min}$ & obtained \\
$\rho_{q}$ & $p 53_{p}$ permeability coefficient & $10 \mu \mathrm{m} / \mathrm{min}$ & obtained \\
\hline
\end{tabular}

Table 2: Parameter values for the 1-dimensional and 2-dimensional model. The permeability coefficients are obtained by numerical data fitting. A more accurate description of the choice of the permeability parameter is done in section 4.4.2.

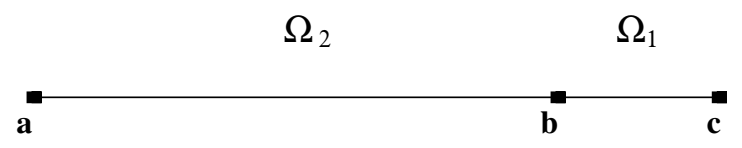

Figure 4: A 1-dimensional simplified domain of the cell.

with with the ODE case. The protein flux from one compartment to the other is not given, as in the ODE case, by an average of the total concentration over the whole compartment, but by the protein concentration that actually exists at the membrane location. In order to test whether the system reproduces the oscillatory behaviour observed in the ODE case, first we analyse it by following the variations of the $\overline{A T M}$ parameter. As explained above, this parameter is meant to represent the DNA damage and it is the switch that turns on the system, giving rise to robust oscillations. In the sequel we fix a time duration of $500 \mathrm{~min}$ to compare the results for different values of the parameters. We observed, see Figure 5(a), that for values of $\overline{A T M}<2$, first the system tends towards a stable equilibrium, then starts to produce damped oscillations (Figures 5(c) and 5(e)). Oscillations become undamped and their amplitude rises when $\overline{A T M}>2$, see Figures $5(\mathrm{~d})$, $5(\mathrm{f})$. As for the ODE case, oscillations disappear again for values of $\overline{A T M}$ higher than a second threshold $(\sim 45)$. It is known from in vitro observations $[2,46]$ that the period of oscillations is stable over different irradiation doses and consequent damages. The same behaviour may be observed on our simulation results that show that once the undamped oscillations occur, the period varies between 26 and 40 minutes. See Figure 5(b) where we plotted the number of peaks of the sustained oscillations observed in $500 \mathrm{~min}$, against $\overline{A T M}$ variations. 


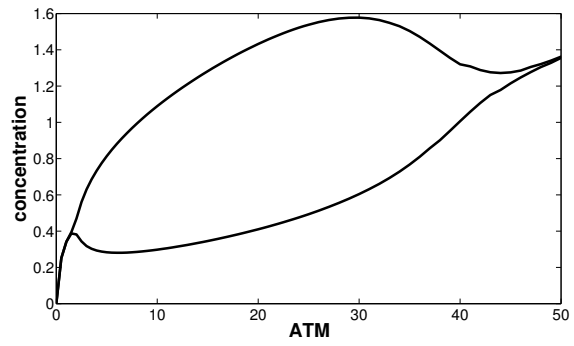

(a) amplitude of oscillations of $q^{(n)}$

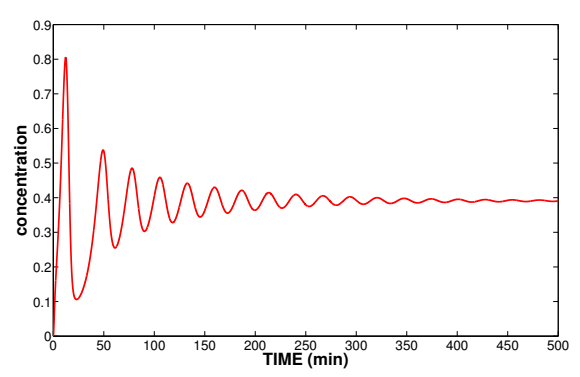

(c) Nuclear $p 53$ over time, $\overline{A T M}=1.5$

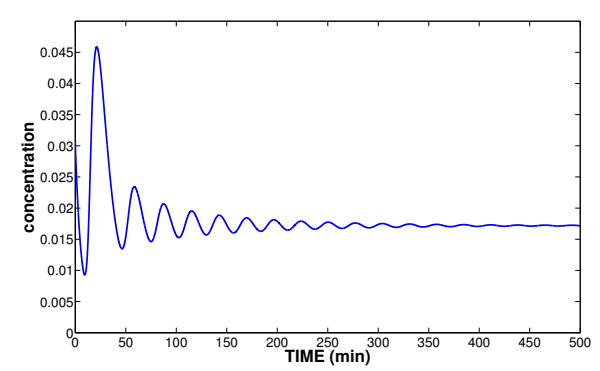

(e) Nuclear $M d m 2$ over time, $\overline{A T M}=1.5$

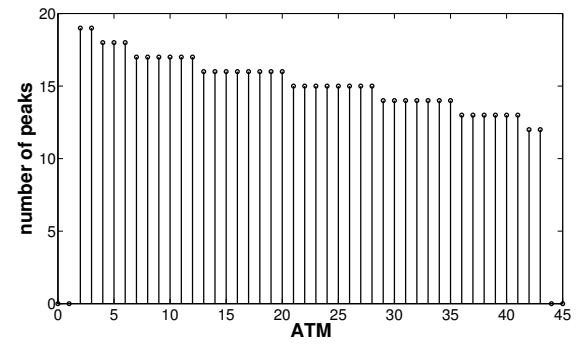

(b) Number of observed peaks in 500 min

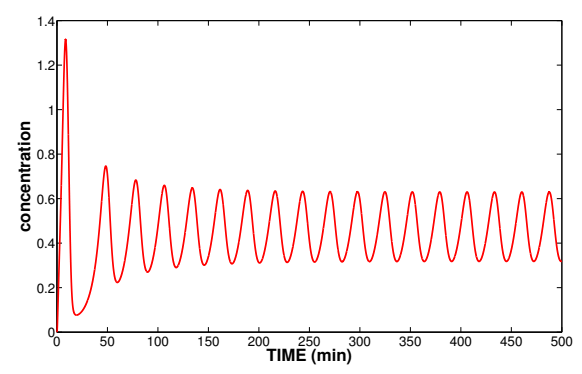

(d) Nuclear $p 53$ over time, $\overline{A T M}=3$

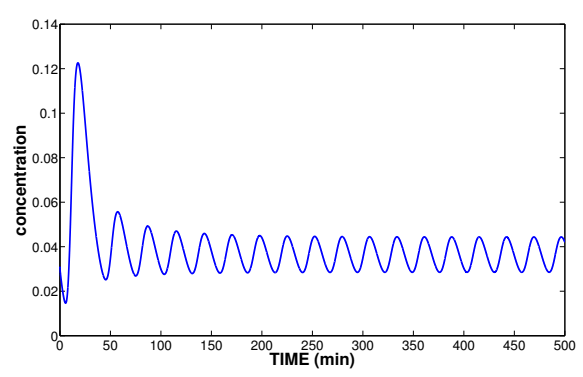

(f) Nuclear $M d m 2$ over time, $\overline{A T M}=3$

Figure 5: 1-dimensional PDE model. (a): amplitude of oscillations of $q^{(n)}$ as a function of $\overline{A T M}$. (b): Number of sustained oscillations peaks observed in a fixed duration of 500 minutes. Oscillations in the 1-dimensional case: (c),(d) evolution of p53 concentration, nuclear compartment. (e), (f): evolution of Mdm2 concentration, nuclear compartment. Undamped oscillations occur only for value of $\overline{A T M}>2(A T M=0.2)$.

4.3.1. The response of the system is robust upon changes of the diffusion coefficients.

Once the oscillatory regime is established, we can vary the spatial parameters, namely diffusion and permeability, to understand how the system responds to spatial perturbations. In Section 4.2 we pointed out that the physiological ratio between protein and mRNA diffusion is about 100:1. Keeping this ratio fixed, we 


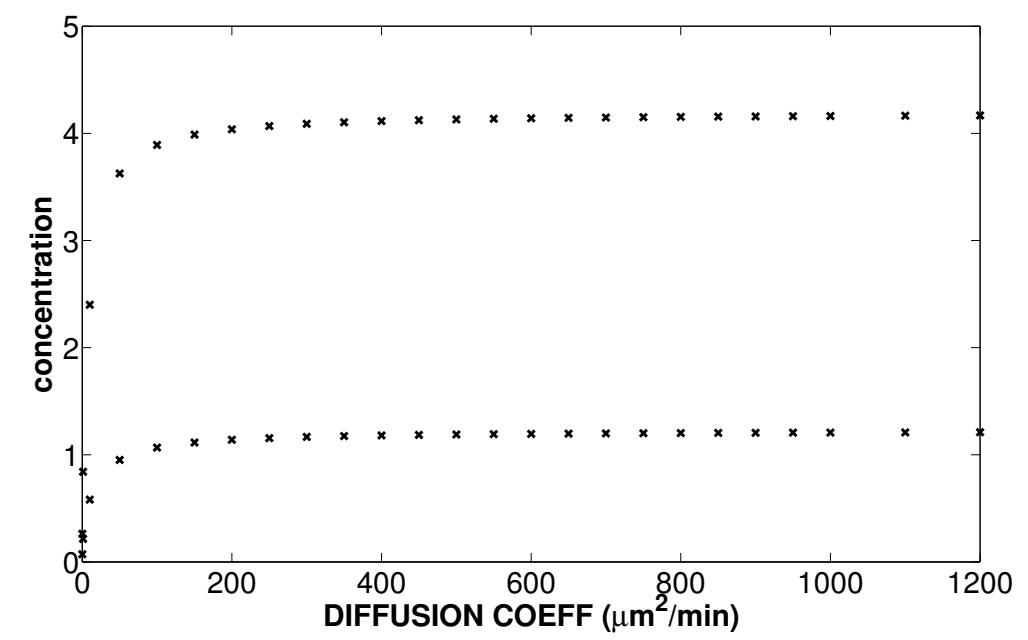

(a)

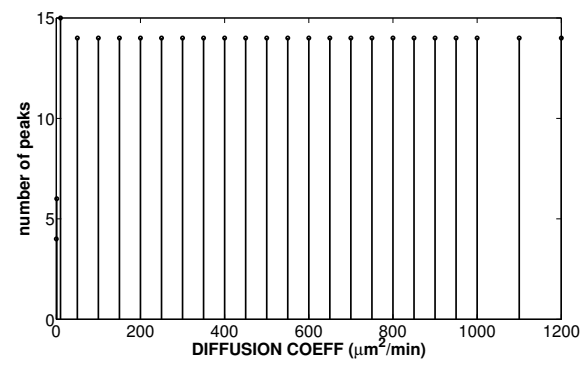

(b)

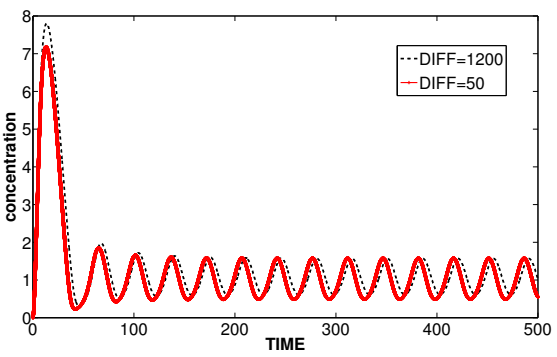

(c)

Figure 6: Oscillations in the 1-dimensional PDE case. If the ratio between protein to mRNA diffusion is kept 100:1, varying the diffusion coefficient does not compromise the oscillatory behaviour of the system. (a): amplitude of oscillations of $\bar{q}^{(n)}$ over the diffusion coefficients. (b): Number of peaks of the sustained oscillations during $500 \mathrm{~min}$ for each diffusion coefficient value. (c): Evolution of $q^{(n)}$ over time, for a diffusion coefficient equal to 50 and $1200 \mu \mathrm{m}^{2} / \mathrm{min}$.

performed simulations, varying only the diffusion coefficients. Our results show that the oscillatory behaviour is highly robust over a wide range of variation for the diffusion coefficient. Oscillations arise for low diffusion coefficient (see Fig. 6 ) and remain active for very large values of the parameters. The amplitude of oscillations decreases slightly with the diffusion coefficient but the period of oscillations is almost constant. We can observe damped oscillations for values of protein diffusion lower than $10 \mu \mathrm{m}^{2} / \mathrm{min}$, but once this threshold is crossed, undamped oscillations arise with a period of about 35 minutes (lower than the period observed in biological experiments $[2,46])$. Conversely, if simulations are performed with a 1:1 ratio between mRNA and protein diffusion, oscillations disappear quickly, for 
diffusion values $D \geq 100$, as shown in Figure 7 . This suggests that the difference between mRNA and protein diffusion coefficients is crucial to the oscillations of the system.

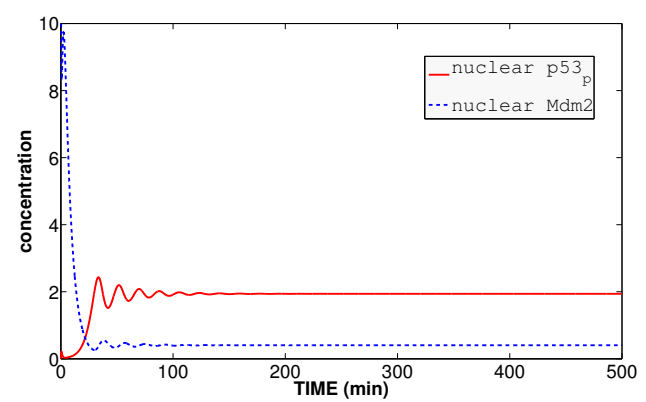

Figure 7: 1-dimensional PDE model. Temporal evolution of nuclear $p 53_{p}$ and $M d m 2$ concentrations. For homogeneous values of the diffusion coefficient (ratio 1:1) only damped oscillations occur. Here we fixed $D_{s}=200$, for $s=p, m, r, q$. All the other parameter values are fixed to the reference ones reported in Table 1 and 2 .

\subsubsection{Low permeability is essential for oscillations in the 1-D case.}

We analysed the behaviour of the system under variations of the permeability coefficient. In order to cross the nuclear membrane, proteins and RNAs need to pass through large protein channels, called nuclear pore complexes (NPCs), that let only authorised molecules to pass through. Even though a nuclear pore can be up to $100 \mathrm{~nm}$ long [53] and the access to the pore is highly controlled, the translocation pathway is impressively efficient. The mass flow through a single NPC can be up to $80 \mathrm{MDa} / \mathrm{s}$ [54] and the time needed for translocation through the pore lasts only $5-7 m s$ [55]. In order to understand whether the permeability parameter is determinant for the model behaviour, we performed numerical simulations for different permeability values. As in Section 4.3.1, the ratio between the diffusion coefficients of protein and mRNA has been set to 100:1. In the 1-dimensional case, undamped oscillations occur for values of the permeability $\rho_{s}$ included between 3 and $20 \mu \mathrm{m} / \mathrm{min}$. Some damped oscillations still appear for values of the parameter $\rho_{s} \in[20,40]$, as shown in Figure 8. We also notice that, unlike in the previous experiments on diffusion coefficients, the period of the oscillations depends highly on permeability variations. Figure $8(\mathrm{~b})$ shows the number of peaks of p53 level observed in 500 minutes.

These first results bring out the importance of the spatial variable: analysing variations of the system with respect to coefficients that could not be studied in the ODE case, we have pointed out the strong dependence of the solution upon the permeability coefficient. However, the range of values over which we can observe the expected oscillatory behaviour is lower than the permeability values proposed 


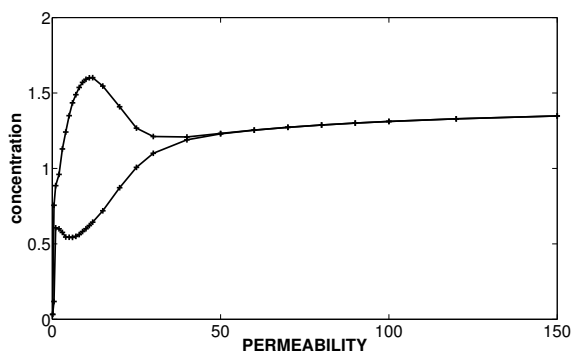

(a)

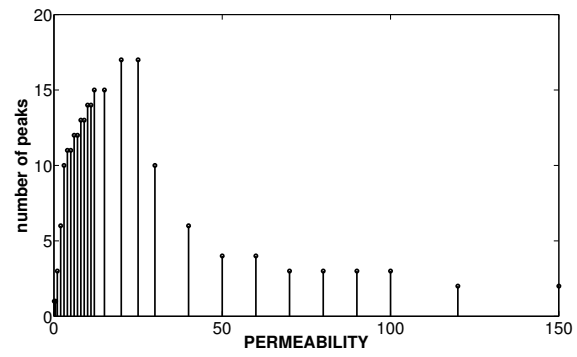

(b)

Figure 8: 1-dimensional PDE model. (a): Amplitude of oscillations of $\bar{q}^{(n)}$ concentration, for non homogeneous permeability coefficient: protein permeability:RNA permeability fixed to 100:1. All the other parameter values are fixed to the reference ones reported in Table 1 and 2. (b) Corresponding number of oscillations in $500 \mathrm{~min}$.

in the literature (see e.g. [30]), that range around $100 \mu \mathrm{m} / \mathrm{min}^{-1}$. We also noticed that different diffusion coefficients did not influence - neither qualitatively nor quantitatively - the behaviour of the system. Since the 1-dimensional case is an oversimplified model of the cell, where the nuclear membrane is reduced to a single point and diffusion is too fast to allow for significantly different behaviours, we will now analyse the behaviour of the model in a still simple, but more realistic, 2-dimensional domain.

\subsection{Results in the 2-Dimensional domain}

In this section we analyse the system in a 2-dimensional cell-shaped domain. All simulations have been performed using the open source tool FreeFem $++^{1}$. For our simulations we chose the domain represented in Fig. 9, where the total area is of about $300 \mu^{2}$, while the ratio between cytoplasmic and nuclear areas is $10: 1$. These values will be varied only when specified. References values for spatial parameters have been reported in Table 2. Most importantly, we remark that all the following simulations are done for the value of $A T M=3$, for which sustained oscillations occur. As we verified by numerical simulations, also in the 2-dimensional case, sustained oscillations occur for values of $A T M$ higher than a minimum threshold and disappear if the value of $A T M$ is too high.

We reproduced the spatial dynamics of the p53-Mdm2 system, as observed in $[2,46]$. As can be observed in Figure 10, 30 min after damage sensing, the p53 protein accumulates in the nucleus. A first peak of p53 concentration appears 1

\footnotetext{
${ }^{1}$ FreeFem ++ (http://www.freefem.org $/ \mathrm{ff}++/$ ) is a computer language dedicated to the finite element method, developed at Jacques-Louis Lions Laboratory, Pierre et Marie Curie University (UPMC), Paris.
} 


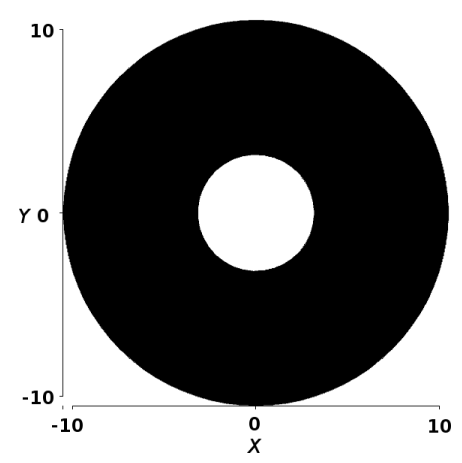

Figure 9: Simulation domain of the 2-dimensional PDE model, Volume ratio: $(C: N)=10: 1$

hour after the start of simulations. Then the cytoplasm and the nucleus empty and a second cycle starts. Oscillations of Mdm2 follow, see Figure 11. The period of oscillations is about $300 \mathrm{~min}$ and corresponds to the actual period experimentally observed $[2,46]$.
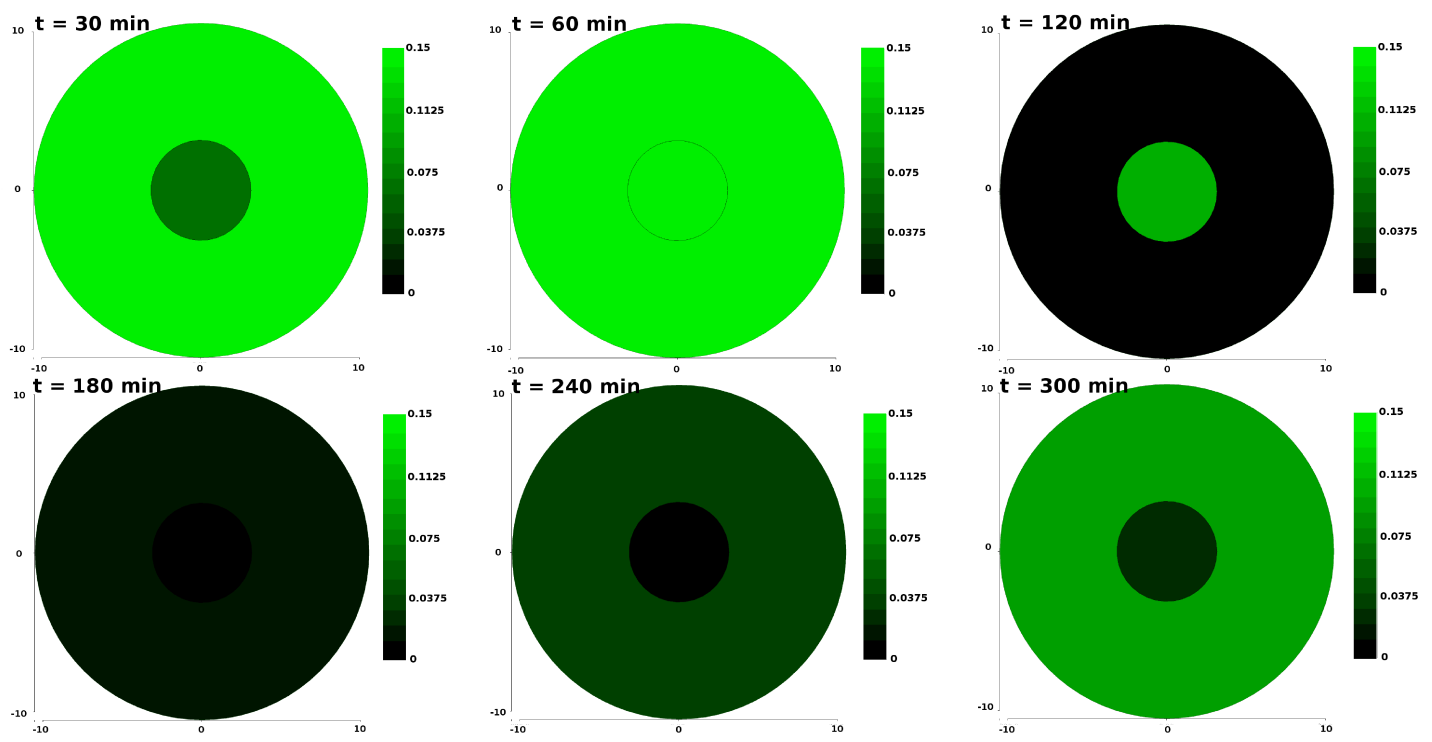

Figure 10: 2-dimensional PDE model. Spatial distribution of $p 53_{p}$ at different time-step on the reference domain. Phosphorylated p53 spatial distribution and temporal evolution. As observed in biological experiments $[2,46]$ p53 accumulates in the nucleus. The peak of p53 protein is observed at $1 \mathrm{~h}$ from damage sensing. Then the level of p53 steps back towards the initial state.

\subsubsection{Oscillations exist for realistic protein and $m R N A$ diffusion values.}

Following the results of $[48,49]$ and the numerical results of the previous section, we fixed the protein to mRNA diffusion coefficient ratio to $100: 1$. As in 

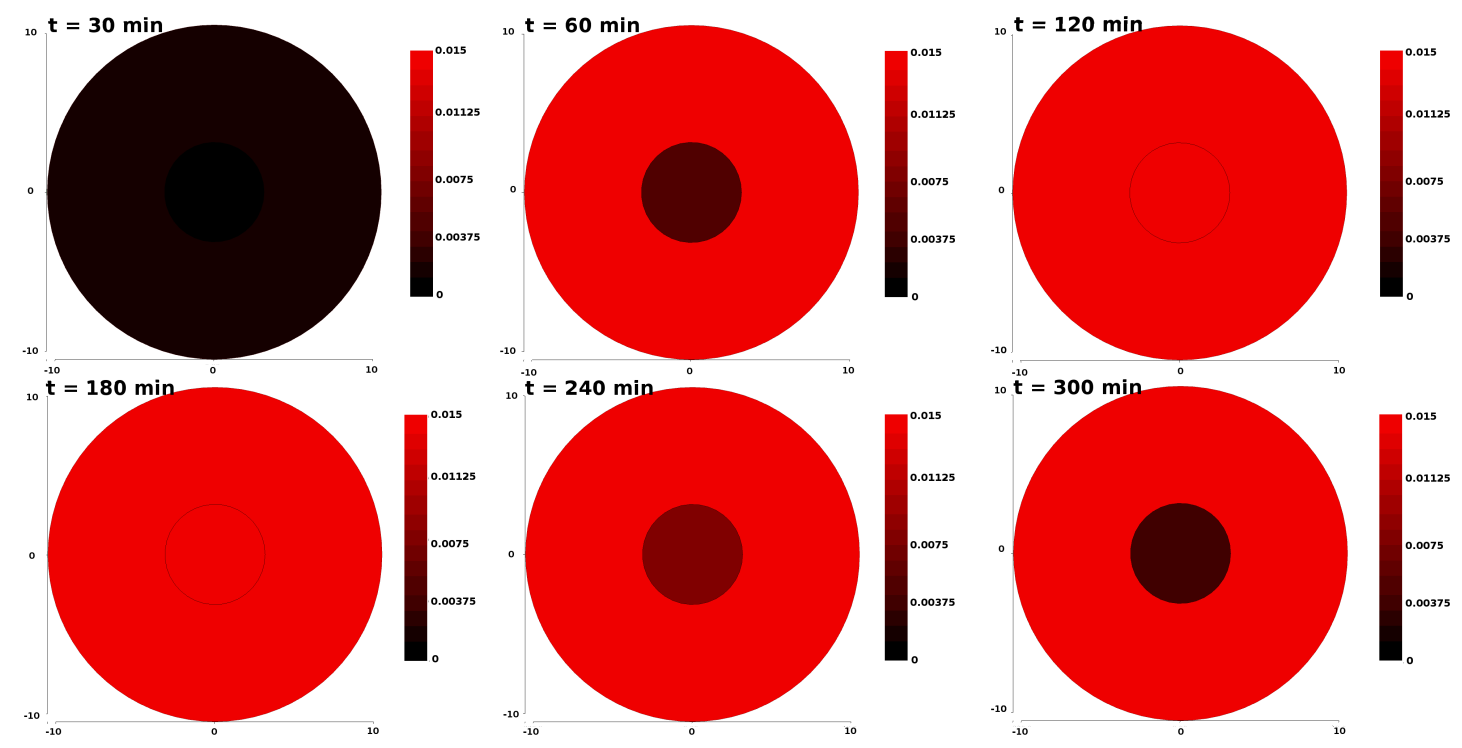

Figure 11: 2-dimensional PDE model. Mdm2 evolution in time and space. As shown in biological experiments, the peak level of Mdm2 follows the peak of p53 [46]. The peak of nuclear Mdm2 takes place at $\mathrm{t}=2 \mathrm{~h}$, while p53 peaks at $\mathrm{t}=1 \mathrm{~h}$. To compare with p53 evolution, see Figure 10 .

section 4.3.1, we observe a robust oscillatory behaviour, with sustained oscillations occurring for diffusion values ranging in $[10,1000] \mu \mathrm{m}^{2} / \mathrm{min}$ (see Table 3 and Figure 12). Comparing these results with the 1-dimensional case, we remark that diffusion values play a more important role. Indeed in Section 4.3.1 we obtained an oscillatory behaviour for all diffusion values $>10 \mu \mathrm{m}^{2} / \mathrm{min}$. Adding the second dimension to the system, we remark instead that oscillations disappear for values of the diffusion coefficient higher than $1000 \mu \mathrm{m}^{2} / \mathrm{min}$, which implies that very fast diffusion of molecules prevents the occurrence of oscillations. This emphasizes the importance in the 2D model of spatial diffusion, with physiological values for the diffusion cefficients. As shown in [51] experimentally observed p53 mobility reduces drastically after DNA damage, and its diffusion coefficient passes from $\sim$ $18 \mu \mathrm{m}^{2} / \mathrm{s}$ to $\sim 3 \mu \mathrm{m}^{2} / \mathrm{s}\left(1000 \mu \mathrm{m}^{2} / \mathrm{min}-180 \mu \mathrm{m}^{2} / \mathrm{min}\right)$, within 8 hours. This reduced mobility is probably due to increased protein-protein interactions and DNA binding of active p53. Interestingly, the oscillatory dynamics is captured by our model for all those different values. Nevertheless, we did not consider the diffusion of p53 as a function of time and DNA damage, which is an open option left for future works.

\subsubsection{Period depends on permeability, but sustained oscillations are always present.}

We tested again the robustness of the system towards perturbations of the permeability coefficients. We know, from the previous section, that in the 1dimensional case the response of the system is sensitive to permeability variations. 


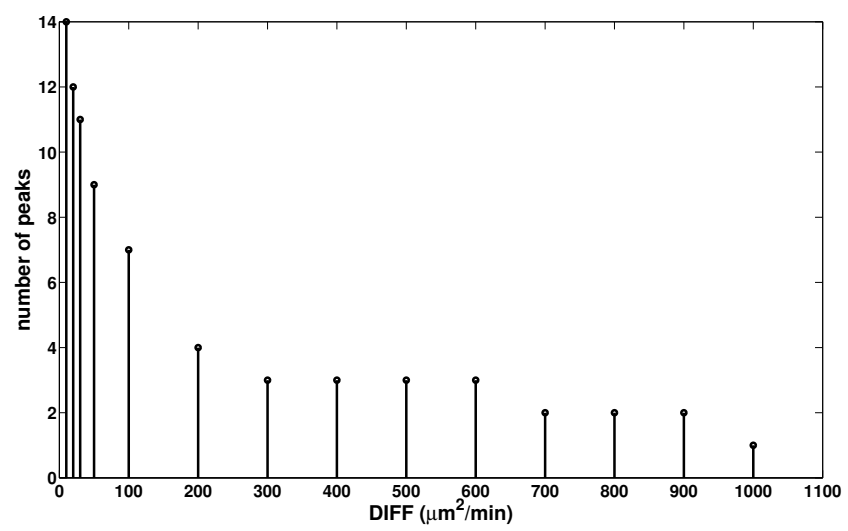

Figure 12: 2-dimensional PDE model. Number of peaks of sustained oscillations observed in the fixed time laps of $500 \mathrm{~min}$. On the $\mathrm{x}$-axis the values of the corresponding diffusion coefficient expressed in $\mu m^{2} / \mathrm{min}$.

In the 1-dimensional setting, oscillations disappear for permeability values lower than the realistic ones (see [30]). In the 2-dimensional model the system reacts in a different way, and we can observe oscillations over a much larger range of the permeability coefficient. We set the diffusion constant to $600 \mu \mathrm{m}^{2} / \mathrm{min}$ for proteins and to $6 \mu \mathrm{m}^{2} / \mathrm{min}$ for the variable $r$, representing the mRNA, and we varied the permeability coefficient.

In the 2-dimensional case the system is much more robust and oscillations arise for permeability values strictly higher than $5 \mu \mathrm{m} / \mathrm{min}$. We notice that the amplitude of oscillations is almost constant (see Figure 13(a)), whereas, again, it is the period of oscillations that exhibits the most remarkable variations. It varies from $250 \mathrm{~min}$ for permeability values of $10 \mu \mathrm{m} / \mathrm{min}$ to about $40 \mathrm{~min}$ when the permeability is set to $200 \mu \mathrm{m} / \mathrm{min}$ (and $2 \mu \mathrm{m} / \mathrm{min}$ for the mRNA), see Fig 13(b) and Table 3.

We notice that, in the 2-dimensional setting, the period of oscillations strongly depends on these spatial coefficients. For high permeability constants the frequencies of oscillations are high, while for lower permeabilities we can reproduce the period of in vitro observations (about 4-5h, [2]).

The discrepancy between our values and those found in the literature could be due to the simplification of the transport machinery used in our model. We supposed indeed that proteins could traverse the nuclear membrane by themselves, which is not the case. Proteins like p53 or Mdm2 need to be carried by a chaperone through the membrane and then be released in the destination compartment. The translocation process imply a number of reactions, like the recognition by the importin and exportin proteins and the binding (and unbinding) to the small GTPase RAN, that handle the directionality of the transport. The time needed 


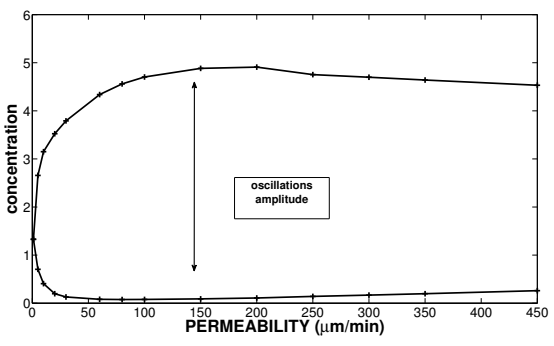

(a) Amplitude of oscillations

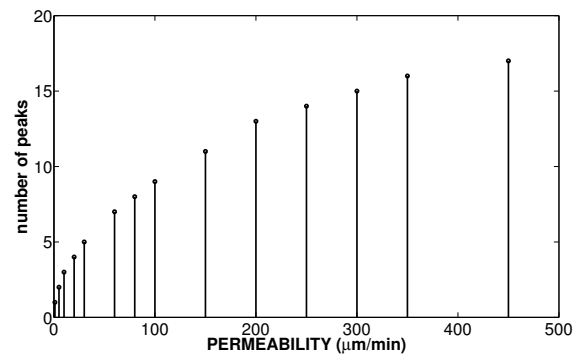

(b) Number of oscillations in 500 minutes

Figure 13: 2-dimensional PDE model. Oscillations in the 2-dimensional case. Variations according to the permeability coefficient: in the 2-dimensional domain, undamped oscillations appear for a wide range of permeability values (see Table 3 ). In (a) we plotted the amplitude of oscillations for $q^{(n)}$, while in (b) we plotted the number of oscillations in $500 \mathrm{~min}$. The protein to mRNA permeabilty ratio is fixed to $100: 1$.

\begin{tabular}{|c|c|c|c|}
\hline Parameter & Description & Ref. values & values for oscillations \\
\hline$V o l$ & Total area of the simulations domain & $300 \mu m^{2}$ & $V o l>0\left(\mu m^{2}\right)$ \\
$V_{r}$ & Volume ratio Cytoplasm:Nucleus & 10 & $2 \leq V_{r} \leq 100$ \\
$\rho_{s}$ & Protein permeabilities & $10 \mu \mathrm{m} / \mathrm{min}$ & $5 \leq \rho_{s} \leq 5000(\mu \mathrm{m} / \mathrm{min})$ \\
$D_{s}$ & Protein diffusion coefficients & $600 \mu \mathrm{m}^{2} / \mathrm{min}$ & $10 \leq D_{i} \leq 1000\left(\mu \mathrm{m}^{2} / \mathrm{min}\right)$ \\
\hline
\end{tabular}

Table 3: Parameter ranges of spatial values for which oscillations occurs. Remark: As explained within the text, the ratio "protein diffusion:mRNA diffusion" has been fixed to 100:1. Consistently to this choice also the ratio "protein permeability:mRNA permeability" has been fixed to 100:1. Range of parameters obtained as in Table 1.

for these events to occur is not taken into account by our model and this led us to set low permeability values, namely $10 \mu \mathrm{m} / \mathrm{min}$ instead of $100 \mu \mathrm{m} / \mathrm{min}$ [30], in order to get oscillations with the period observed in the literature. Note that this point is more extensively commented on Section 6 .

\subsubsection{Period depends on the total volume of the cell rather than on cytoplasmic to nuclear volume ratio.}

The spatial treatment of the problem allowed us to analyse the response of the model in different domains. Numerical simulations have been performed over a large range of domains having different total area, while the ratio between cytoplasmic and nuclear area was kept fix at $10: 1$. Our results show that the oscillatory dynamics is the constant response of the system. However the period of oscillations depends on the total volume of the in silico cell, see Fig. 14(b). For smaller volumes, the period is shorter and it rises with the volume. We observed variations between 55 and $1000 \mathrm{~min}$.

We also analysed how the nuclear:cytoplasmic volume ratio affects the response 
of the system. In this case, we fixed the total area to $300 \mu m^{2}$ (Figure 9), and we varied the nuclear area. Here again, the temporal dynamics of the system is oscillatory and oscillations do not depends on the volume ratio. The period of sustained oscillations varies between 175 and $400 \mathrm{~min}$. Two or three peaks of p53 level (in the fixed time lapse of 500 minutes) can be observed in Figure 15(b) for volume ratios varying between 2 and 100. See Figures 14 and 15 below and Table 3 for more quantitative details.

One can remark that the dynamics of the system is robust as sustained oscillations can be observed over different domains. This information let us speculate that the role of oscillations is crucial for the p53-Mdm2 system. Indeed, even if we change drastically the physical environment, or the cell shape (as we will see in the next section), sustained oscillations are always present.

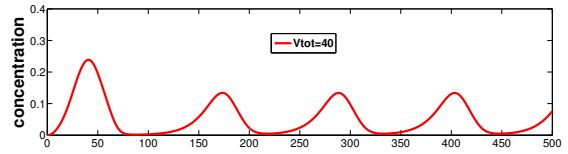

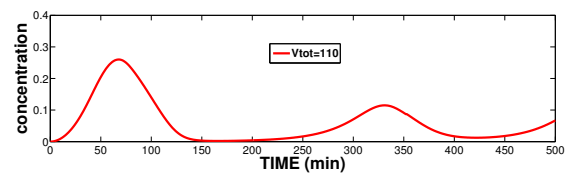

(a)

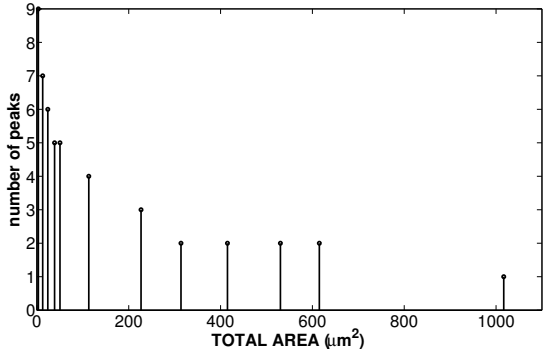

(b)

Figure 14: 2-dimensional PDE model. (a): Oscillations of nuclear $p 53_{p}$ (average concentrations) for different total volume $\left(V_{t o t}\right)$ and fixed volume ratio $\left(V_{r}\right)$. (b): number of oscillations occurring in 500 min for different total volumes.

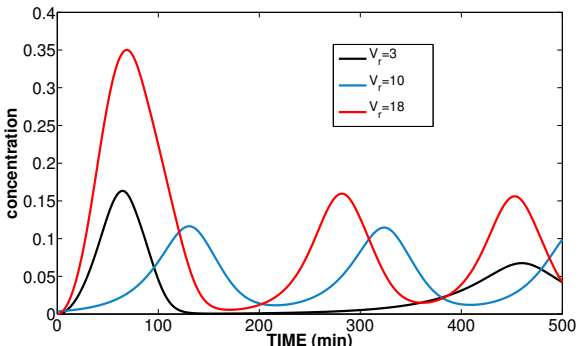

(a)

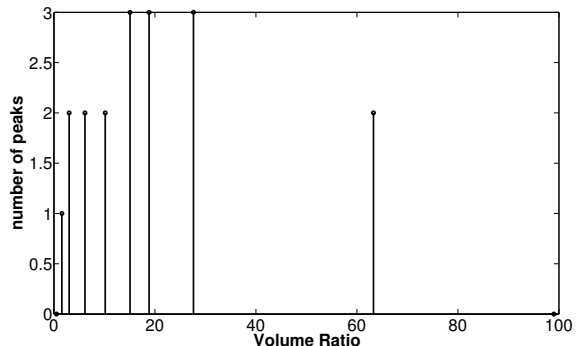

(b)

Figure 15: 2-dimensional PDE model. (a) Oscillations of nuclear p53 (average concentrations) for different volume ratios $\left(V_{r}\right)$ and fixed total area, $V_{r}=\frac{\text { cytoplasmic area }}{\text { nuclear area }}$. (b): number of oscillations occurring in $500 \mathrm{~min}$ for different volume ratio. 


\subsubsection{The geometry of the domain does not change the dynamics of the system.}

We analysed the behaviour of the system over different geometric domains: rectangular, elliptic and 'cell-shaped' domains. In accordance with Terry et al [56] we conclude that the geometry of the domain does not influence the dynamic response of the system. In Figure 16, we reported the cellular domain used in simulations and the corresponding temporal evolution of nuclear and cytoplasmic levels of p53, all three domains having approximately the same area and same volume ratio. As can be seen, the reproduced temporal dynamics of the three systems are the same. Furthermore, the spatial dynamics does not depend on the shape of the domain, as can be observed in Figure 17 where we reported the simulations results in two different domains.

We also performed simulations locating the ribosomes, the big protein complexes, scattered within the cytoplasm, that translate the mRNAs into proteins. We designed the spatial distribution of ribosomes in such a way that their total concentration did not change, varying only their location. To do this, we considered different functions defined on the domain of Figure 9, all having the same mean. No influence of the ribosome location could be observed and we could conclude that the oscillatory behaviour is robust upon changes on the location of ribosomes. This result differs from [27] where the authors stated that the p53 oscillatory response was dependent on ribosome location. However the same authors in [28], when more precisely studying the spatial distribution of ribosomes, observed a lower influence of this location. In an equivalent manner, we changed the transcription locus in the nucleus, where the mRNA of Mdm2 is produced. We fixed the mean of the function representing the transcription domain and we varied its location within the nucleus. Also in this case, we could not observe any quantitative or qualitative variation of the cell behaviour.

\section{Summary of the results}

In this work we studied a new model for p53 which describes both its temporal and spatial dynamics. Considering only the basic pathways and the distinction between nucleus and cytoplasm we have reproduced the spatial and temporal oscillations of the p53-Mdm2 system. Firstly, we have shown that we could reproduce sustained oscillations for the p53-Mdm2 network by considering a nuclear and a cytoplasmic compartment, without further introduction of intracellular space, in Section 3.1. Next we studied a PDE model by introducing a spatial variable in the previously designed ODE model. We were able to reproduce oscillations with the period observed in in vitro experiments, namely $4-5$ hours [2], using realistic diffusion coefficients, by this addition of a spatial variable. Furthermore we observed that the response of the system strongly depends on the spatial coefficients of the system (diffusion, permeability, total volume of the cell), which points out 

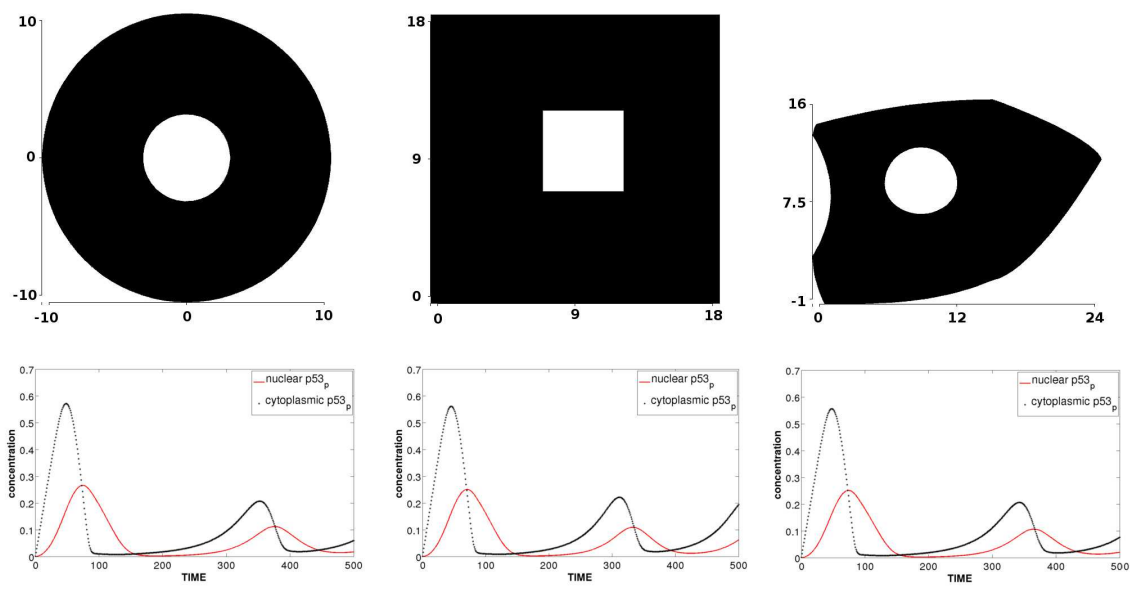

Figure 16: 2-dimensional PDE model. Different simulation domains having the same total volume and same nucleus to cytoplasmic volume ratio, lead to similar oscillatory behaviours of nuclear and cytoplasmic concentration of phosphorylated p53.

the significant role of space in the p53 system. Moreover by means of the PDE model, we have also shown that, using realistic diffusion coefficients for protein and mRNA [48, 49, 50, 51], it is possible to reproduce the oscillatory p53 regime with the exact observed period of oscillations, and that these oscillations are present for all known values of p53 diffusivity [51] (knowing that p53 mobility is reduced after DNA damage).

We have also studied how the system responds to variations in permeability coefficients, total volume and nuclear to cytoplasmic volume ratio. We have shown that the oscillatory response is robust towards such variations, and that the period of oscillations depends on the permeability coefficient, and also on the total volume of the cell.

The distinction between nucleus and cytoplasm is the characteristic feature of eukaryotic cells. We think that a model of signal transduction needs to consider this basic and simple distinction in order to be consistent with common knowledge of the intracellular biology and topology.

\section{Conclusions and Perspectives}

We have proposed in this paper a model for p53 nuclear accumulation. We have shown that the negative p53-Mdm2 feedback reproduces the oscillatory behaviour observed in cultured cells, if the distinction between the nuclear and cytoplasmic compartments is taken into account. This implies locating the main cellular processes and making explicit the delays due to transcription, translation and translocation between compartments. Firstly, we have studied the response of the ODE 

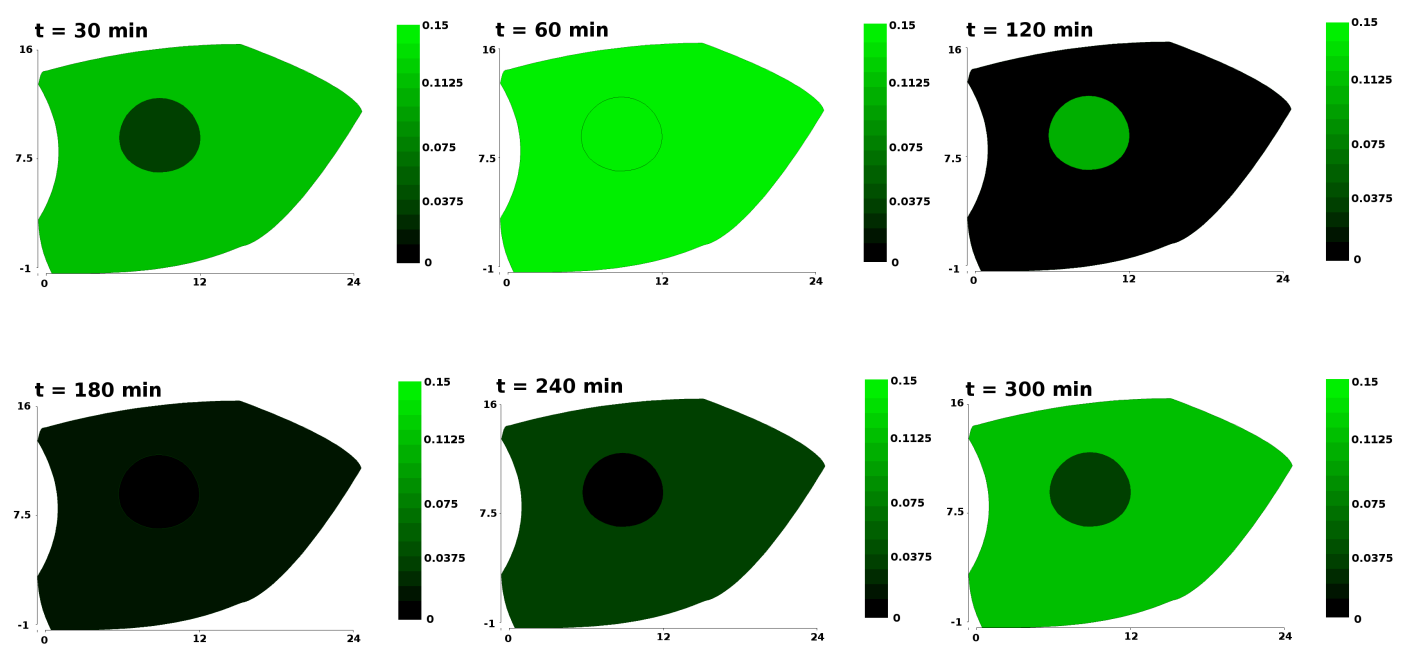

(a)
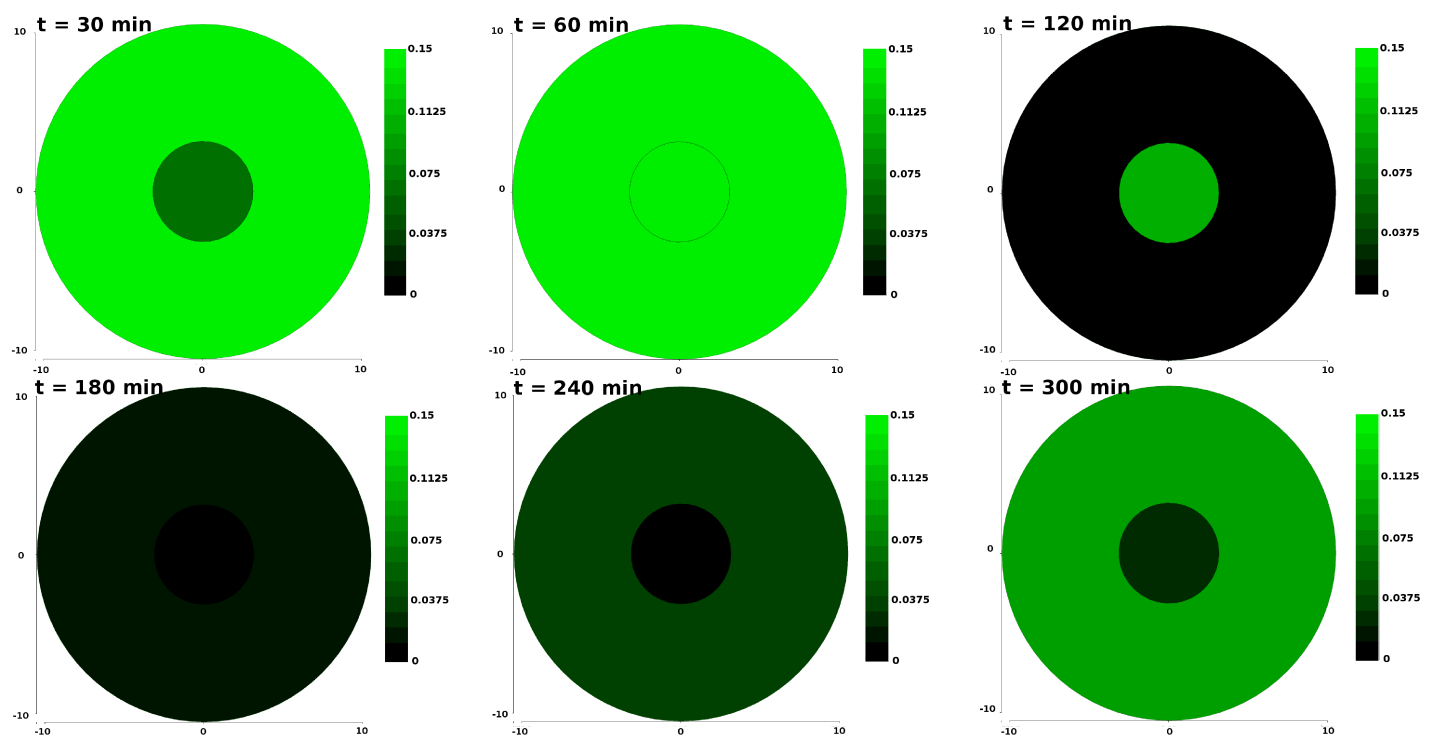

(b)

Figure 17: 2-dimensional PDE model. (a): p53 oscillations in the 2-dimensional case: after 30 minutes the concentration of the molecule accumulates in the whole cell. Then the nucleus fills up and the cytoplasmic concentration decreases. The cycle starts again. (b): spatial dynamics of p53 in the reference domain. The spatial and temporal dynamics do not depend on cell shape. 
differential system, and we have verified that the dynamics of the network proposed was the expected one. We have reproduced undamped oscillations against ATM variations showing the existence of a Hopf supercritical bifurcation. Then we have introduced a spatial variable and numerically analysed the simulation results in the new PDE setting. Our choice to develop a spatial model of the p53 network has led us to remark that taking into account physiological phenomena within the cellular space explains in biologically relevant details the expected oscillations. We have shown that the experimental diffusion values fit our model and reproduce the oscillatory behaviour with a good estimation of the period observed in vitro. We have provided evidence that the oscillatory behaviour of the system is also robust towards variations of the nuclear to cytoplasmic ratio and of cell shapes. This allows us to speculate that such a robust response of the system towards changes in physical coefficients, as cell volume, diffusion coefficient or volume ratios, testifies the crucial role of oscillations in the p53 system.

It is worth noting that the permeability values that reproduce the physiological behaviour in our model are lower than the values proposed in other works [29, 30]. We believe that, taking into account more signalling pathways involved in the import and export nucleocytoplasmic machinery should permit to consider more realistic permeability values. Indeed, it is known that the translocation rate is given by the nucleocytoplasmic transport machinery [50], and not by the translocation through the nuclear pore complexes, since the time during which molecules bind to the central region of the nuclear pore is very low [55]. Thus to take into account more realistic transport timing it would be important to couple the model we have designed with the model of nucleocytoplasmic transport studied in [29]. Preliminary studies with compartmental models are under way. It would also be interesting to model the cytoskeloton activity in the transport mechanism, as done in $[28,29]$. It has indeed been shown that p53 uses the microtubule filaments in order to get to the nucleus faster [57, 58]. However, there is still no experimental evidence for a role of microtubules in Mdm2 cytosolic transport. Another important extension of our model, that would introduce more realism, would be to include the third dimension and explore the behaviour of the system in the 3 -dimensional setting.

To conclude on a medically oriented note, the simplicity of the network considered in this work may offer in the future an effective tool to understand the effects of known mutations of p53 with respect to the different mechanism that we represent in our model, as nuclear import, translation, or phosphorylation. Indeed p53 is known to be mutated in more than $50 \%$ of cancer cells and it is a future goal with possible pharmacological and clinical consequences for us to understand how these mutations influence the spatio-temporal dynamics of p53. 


\section{Acknowledgements.}

The authors are gratefully indebted to Frédérique Billy for her time spent on reading the manuscript and suggesting improvements to it, and to Robin Fåhraeus for his useful comments on the model from an expert biologist's point of view.

[1] R. L. Bar-Or, R. Maya, L. A. Segel, U. Alon, A. J. Levine, M. Oren, Generation of oscillations by the p53-mdm2 feedback loop: a theoretical and experimental study., Proc Natl Acad Sci U S A 97 (21) (2000) 11250-11255. doi:10.1073/pnas.210171597.

URL http://dx.doi.org/10.1073/pnas.210171597

[2] G. Lahav, N. Rosenfeld, A. Sigal, N. Geva-Zatorsky, A. J. Levine, M. B. Elowitz, U. Alon, Dynamics of the p53-mdm2 feedback loop in individual cells., Nat Genet 36 (2) (2004) 147-150. doi:10.1038/ng1293.

URL http://dx.doi .org/10.1038/ng1293

[3] S. Fang, J. P. Jensen, R. L. Ludwig, K. H. Vousden, A. M. Weissman, Mdm2 is a ring finger-dependent ubiquitin protein ligase for itself and p53., J Biol Chem 275 (12) (2000) 8945-8951.

[4] N. D. Marchenko, W. Hanel, D. Li, K. Becker, N. Reich, U. M. Moll, Stress-mediated nuclear stabilization of p53 is regulated by ubiquitination and importin-alpha3 binding., Cell Death Differ 17 (2) (2010) 255-267. doi:10.1038/cdd.2009.173.

URL http://dx.doi.org/10.1038/cdd.2009.173

[5] D. Michael, M. Oren, The p53-mdm2 module and the ubiquitin system., Semin Cancer Biol 13 (1) (2003) 49-58.

[6] K.-X. Shu, B. Li, L.-X. Wu, The p53 network: p53 and its downstream genes., Colloids Surf B Biointerfaces 55 (1) (2007) 10-18. doi:10.1016/j.colsurfb.2006.11.003.

URL http://dx.doi.org/10.1016/j.colsurfb.2006.11.003

[7] D. A. Freedman, L. Wu, A. J. Levine, Functions of the mdm2 oncoprotein., Cell Mol Life Sci 55 (1) (1999) 96-107.

[8] A. M. Carr, Cell cycle. piecing together the p53 puzzle., Science 287 (5459) (2000) 1765-1766.

[9] B. Vogelstein, D. Lane, A. J. Levine, Surfing the p53 network., Nature 408 (6810) (2000) 307-310. doi:10.1038/35042675.

[10] M. F. Lavin, S. Kozlov, Atm activation and dna damage response., Cell Cycle 6 (8) (2007) 931-942. 
[11] F. A. Derheimer, M. B. Kastan, Multiple roles of atm in monitoring and maintaining dna integrity., FEBS Lett 584 (17) (2010) 3675-3681. doi:10.1016/j.febslet.2010.05.031.

[12] S. Saito, A. A. Goodarzi, Y. Higashimoto, Y. Noda, S. P. Lees-Miller, E. Appella, C. W. Anderson, Atm mediates phosphorylation at multiple p53 sites, including ser(46), in response to ionizing radiation., J Biol Chem 277 (15) (2002) 12491-12494. doi:10.1074/jbc.C200093200.

[13] N. D. Lakin, S. P. Jackson, Regulation of p53 in response to dna damage., Oncogene 18 (53) (1999) 7644-7655. doi:10.1038/sj.onc.1203015.

[14] N. C. Reich, M. Oren, A. J. Levine, Two distinct mechanisms regulate the levels of a cellular tumor antigen, p53., Mol Cell Biol 3 (12) (1983) 2143-2150.

[15] M. P. Vierboom, S. Zwaveling, B. GMJ, M. Ooms, G. M. Krietemeijer, C. J. Melief, R. Offringa, High steady-state levels of p53 are not a prerequisite for tumor eradication by wild-type p53-specific cytotoxic t lymphocytes., Cancer Res 60 (19) (2000) 5508-5513.

[16] L. Feng, T. Lin, H. Uranishi, W. Gu, Y. Xu, Functional analysis of the roles of posttranslational modifications at the p53 c terminus in regulating p53 stability and activity., Mol Cell Biol 25 (13) (2005) 5389-5395. doi:10.1128/MCB.25.13.5389-5395.2005.

[17] S. H. Liang, M. F. Clarke, Regulation of p53 localization., Eur J Biochem 268 (10) (2001) 2779-2783.

[18] X. Wu, J. H. Bayle, D. Olson, A. J. Levine, The p53-mdm-2 autoregulatory feedback loop., Genes Dev 7 (7A) (1993) 1126-1132.

[19] Y. Haupt, R. Maya, A. Kazaz, M. Oren, Mdm2 promotes the rapid degradation of p53., Nature 387 (6630) (1997) 296-299. doi:10.1038/387296a0.

[20] B. Novák, J. J. Tyson, Design principles of biochemical oscillators., Nat Rev Mol Cell Biol 9 (12) (2008) 981-991. doi:10.1038/nrm2530.

[21] N. A. M. Monk, Oscillatory expression of hes1, p53, and nf-kappab driven by transcriptional time delays., Curr Biol 13 (16) (2003) 1409-1413.

[22] L. Ma, J. Wagner, J. J. Rice, W. Hu, A. J. Levine, G. A. Stolovitzky, A plausible model for the digital response of p53 to dna damage., Proc Natl Acad Sci U S A 102 (40) (2005) 14266-14271. doi:10.1073/pnas.0501352102. URL http://dx.doi.org/10.1073/pnas.0501352102 
[23] D. A. Ouattara, W. Abou-Jaoudé, M. Kaufman, From structure to dynamics: frequency tuning in the p53-mdm2 network. ii differential and stochastic approaches., J Theor Biol 264 (4) (2010) 1177-1189. doi:10.1016/j.jtbi.2010.03.031.

URL http://dx.doi.org/10.1016/j.jtbi.2010.03.031

[24] A. Ciliberto, B. Novak, J. J. Tyson, Steady states and oscillations in the p53/mdm2 network., Cell Cycle 4 (3) (2005) 488-493.

[25] L. D. Mayo, D. B. Donner, A phosphatidylinositol 3-kinase/akt pathway promotes translocation of mdm2 from the cytoplasm to the nucleus., Proc Natl Acad Sci U S A 98 (20) (2001) 11598-11603. doi:10.1073/pnas.181181198.

[26] K. Gordon, I. van Leeuween, S. Lain, M. Chaplain, Spatio-temporal modelling of the p53-mdm2 oscillatory system, Math. Model. Nat. Phenom. 4(3) (2009) $97-116$.

[27] M. Sturrock, A. J. Terry, D. P. Xirodimas, A. M. Thompson, M. A. J. Chaplain, Spatio-temporal modelling of the hes1 and p53-mdm2 intracellular signalling pathways., J Theor Biol 273 (1) (2011) 15-31. doi:10.1016/j.jtbi.2010.12.016.

[28] M. Sturrock, A. J. Terry, D. P. Xirodimas, A. M. Thompson, M. A. J. Chaplain, Influence of the nuclear membrane, active transport, and cell shape on the hes1 and p53-mdm2 pathways: insights from spatio-temporal modelling, Preprint submitted to Bulletin of Mathematical Biology.

[29] A. Cangiani, R. Natalini, A spatial model of cellular molecular trafficking including active transport along microtubules., J Theor Bioldoi:10.1016/j.jtbi.2010.08.017.

URL http://dx.doi.org/10.1016/j.jtbi.2010.08.017

[30] A. E. Smith, B. M. Slepchenko, J. C. Schaff, L. M. Loew, I. G. Macara, Systems analysis of ran transport., Science 295 (5554) (2002) 488-491. doi:10.1126/science.1064732.

[31] O. Seksek, J. Biwersi, A. S. Verkman, Translational diffusion of macromolecule-sized solutes in cytoplasm and nucleus., J Cell Biol 138 (1) (1997) 131-142.

[32] S. Rajagopalan, F. Huang, A. R. Fersht, Single-molecule characterization of oligomerization kinetics and equilibria of the tumor suppressor p53., Nucleic Acids Res 39 (6) (2011) 2294-2303. doi:10.1093/nar/gkq800. 
[33] L. A. Segel, M. Slemrod, The quasi-steady-state assumption: A case study in perturbation, SIAM Review 31 (3).

URL http://www.jstor.org/stable/2031405

[34] J. S. James P Keener, Mathematical physiology, 2nd Edition, Springer, 1998.

[35] K. M. Dohoney, C. Guillerm, C. Whiteford, C. Elbi, P. F. Lambert, G. L. Hager, J. N. Brady, Phosphorylation of p53 at serine 37 is important for transcriptional activity and regulation in response to dna damage., Oncogene 23 (1) (2004) 49-57. doi:10.1038/sj.onc.1207005.

[36] A. Hill, Proceedings of the physiological society, The Journal of Physiology 40 (1910) i-vii. arXiv:http://jp.physoc.org/content/40/supplement/i.full.pdf + html. URL http://jp.physoc.org/content/40/supplement/i.short

[37] D. P. Xirodimas, C. W. Stephen, D. P. Lane, Cocompartmentalization of p53 and mdm2 is a major determinant for mdm2-mediated degradation of p53., Exp Cell Res 270 (1) (2001) 66-77. doi:10.1006/excr.2001.5314.

[38] D. A. Freedman, A. J. Levine, Nuclear export is required for degradation of endogenous p53 by mdm2 and human papillomavirus e6., Mol Cell Biol 18 (12) (1998) 7288-7293.

[39] J. M. Stommel, N. D. Marchenko, G. S. Jimenez, U. M. Moll, T. J. Hope, G. M. Wahl, A leucine-rich nuclear export signal in the p53 tetramerization domain: regulation of subcellular localization and p53 activity by nes masking., EMBO J 18 (6) (1999) 1660-1672. doi:10.1093/emboj/18.6.1660.

URL http://dx.doi.org/10.1093/emboj/18.6.1660

[40] Y. Zhang, Y. Xiong, A p53 amino-terminal nuclear export signal inhibited by dna damage-induced phosphorylation., Science 292 (5523) (2001) 1910-1915. doi:10.1126/science.1058637.

[41] P. Rangamani, R. Iyengar, Modelling spatio-temporal interactions within the cell., J Biosci 32 (1) (2007) 157-167.

[42] K. B. Wee, U. Surana, B. D. Aguda, Oscillations of the p53-akt network: implications on cell survival and death., PLoS One 4 (2) (2009) e4407. doi:10.1371/journal.pone.0004407.

[43] T. Pu, X.-P. Zhang, F. Liu, W. Wang, Coordination of the nuclear and cytoplasmic activities of p53 in response to dna damage., Biophys J 99 (6) (2010) 1696-1705. doi:10.1016/j.bpj.2010.07.042. 
[44] L. Perko, Differential Equations and Dynamical Systems, 2nd Edition, Springer-Verlag, 1996.

[45] Y. A. Kuznetsov, Elements of Applied Bifurcation Theory, 3rd Edition, Springer-Verlag, 2004.

[46] N. Geva-Zatorsky, N. Rosenfeld, S. Itzkovitz, R. Milo, A. Sigal, E. Dekel, T. Yarnitzky, Y. Liron, P. Polak, G. Lahav, U. Alon, Oscillations and variability in the p53 system, Molecular Systems Biology 2. doi:10.1038/msb4100068.

[47] O. Kedem, A. Katchalsky, Thermodynamic analysis of the permeability of biological membranes to non-electrolytes., Biochim Biophys Acta 27 (2) (1958) 229-246.

[48] D. Fusco, N. Accornero, B. Lavoie, S. M. Shenoy, J. M. Blanchard, R. H. Singer, E. Bertrand, Single mrna molecules demonstrate probabilistic movement in living mammalian cells., Curr Biol 13 (2) (2003) 161-167.

[49] Y. Shav-Tal, X. Darzacq, S. M. Shenoy, D. Fusco, S. M. Janicki, D. L. Spector, R. H. Singer, Dynamics of single mrnps in nuclei of living cells., Science 304 (5678) (2004) 1797-1800. doi:10.1126/science.1099754.

[50] D. Grünwald, R. H. Singer, M. Rout, Nuclear export dynamics of rna-protein complexes., Nature 475 (7356) (2011) 333-341. doi:10.1038/nature10318.

[51] S. Hong, Y.-N. Wang, H. Yamaguchi, H. Sreenivasappa, C.-K. Chou, P.-H. Tsou, M.-C. Hung, J. Kameoka, Measurement of protein 53 diffusion coefficient in live hela cells using raster image correlation spectroscopy (rics)., J Biomater Nanobiotechnol 1 (1) (2010) 31-36. doi:10.4236/jbnb.2010.11004.

[52] W. Hundsdorfer, J. Verwer, Numerical Solution of Time-Dependent Advection-Diffusion-Reaction Equations, Vol. 33 of Springer Series in Computational Mathematics, Springer-Verlag, 2003.

[53] R. Reichelt, A. Holzenburg, E. L. Buhle, M. Jarnik, A. Engel, U. Aebi, Correlation between structure and mass distribution of the nuclear pore complex and of distinct pore complex components., J Cell Biol 110 (4) (1990) 883-894.

[54] K. Ribbeck, D. Görlich, Kinetic analysis of translocation through nuclear pore complexes., EMBO J 20 (6) (2001) 1320-1330. doi:10.1093/emboj/20.6.1320.

[55] T. Dange, D. Grünwald, A. Grünwald, R. Peters, U. Kubitscheck, Autonomy and robustness of translocation through the nuclear pore complex: a singlemolecule study., J Cell Biol 183 (1) (2008) 77-86. doi:10.1083/jcb.200806173. 
[56] A. J. Terry, M. A. J. Chaplain, Spatio-temporal modelling of the nf- $\kappa \mathrm{b}$ intracellular signalling pathway: the roles of diffusion, active transport, and cell geometry., J Theor Biol 290 (2011) 7-26. doi:10.1016/j.jtbi.2011.08.036.

[57] D. M. Roth, G. W. Moseley, D. Glover, C. W. Pouton, D. A. Jans, A microtubule-facilitated nuclear import pathway for cancer regulatory proteins., Traffic 8 (6) (2007) 673-686. doi:10.1111/j.1600-0854.2007.00564.x.

URL http://dx.doi.org/10.1111/j.1600-0854.2007.00564.x

[58] P. Giannakakou, M. Nakano, K. C. Nicolaou, A. O'Brate, J. Yu, M. V. Blagosklonny, U. F. Greber, T. Fojo, Enhanced microtubule-dependent trafficking and p53 nuclear accumulation by suppression of microtubule dynamics., Proc Natl Acad Sci U S A 99 (16) (2002) 10855-10860. doi:10.1073/pnas.132275599.

URL http://dx.doi.org/10.1073/pnas.132275599 